\title{
Lens space surgeries as A'Campo's divide knots
}

\author{
YUICHI YAMADA
}

\begin{abstract}
It is proved that every knot in the major subfamilies of J Berge's lens space surgery (ie, knots yielding a lens space by Dehn surgery) is presented by an L-shaped (real) plane curve as a divide knot defined by A'Campo in the context of singularity theory of complex curves. For each knot given by Berge's parameters, the corresponding plane curve is constructed. The surgery coefficients are also considered. Such presentations support us to study each knot of lens space surgery itself and the relationship among the knots in the set of lens space surgeries.
\end{abstract}

14H50, 57M25; 57M27

Dedicated to Professor Takao Matumoto on the occasion of his 60th birthday.

\section{Introduction}

If $r / s$ Dehn surgery on a knot $K$ in $S^{3}$ yields the lens space $L(p, q)$, we call the pair $(K, r / s)$ a lens space surgery, and we also say that $K$ admits a lens space surgery and that $r / s$ is the coefficient of the lens space surgery. The task of classifying lens space surgeries, especially knots that admit lens space surgeries, has been a focal point in low-dimensional topology and has been invigorated of late by results from the Heegaard Floer homology theories of Ozsváth-Szabó [35] (see also Hedden [26] and Tange [39] and so on). Before the first hyperbolic examples found by Fintushel-Stern [17] in 1980, only torus knots (by Moser [32]) and their 2-cables (by Bailey-Rolfsen [6]) were known. After [17], some more examples were found (see Maruyama [30]). In 1990, Berge [10] pointed out a "mechanism" of known lens space surgery, that is, doubly primitive knots in the Heegaard surface of genus 2. Berge also gave a conjecturally complete list of such knots, described them by Osborne-Stevens" "R-R diagrams" in [34] and classified such knots into three families and 12 types in detail:

(1) Knots in a solid torus (Types I, II, ...,VI)

Dehn surgery along a knot in a solid torus whose resulting manifold is also a solid torus. This family was studied in [11]. 
(2) Knots in genus-one fiber surface (Types VII and VIII)

Dehn surgery along a knot in the genus-one fiber surface (of the right/left-handed trefoil (Type VII) or of the figure eight (Type VIII)); see Baker [7; 9] and Yamada [42].

(3) Sporadic examples (a), (b), (c) and (d) (Types IX, X, XI and XII, respectively)

Their surgery coefficients are also decided. Thus we call them Berge knots of lens space surgery, or Berge lens space surgeries. The numberings VII, ..., XII are also used in the recent works of Baker [8; 9]. Gordon [21;22] conjectured that every knot of lens space surgery is a doubly primitive knot. Berge has claimed that his list of doubly primitive knots is complete (ie, any doubly primitive knot belongs to (1), (2) or (3)), but a proof has not appeared.

In the present paper, we are concerned with the family (1). Its subfamily Type I consists of torus knots. Type II consists of 2-cables of torus knots. Their presentations as A'Campo's divide knots are already studied by Goda, Hirasawa and the author [18] and the author alone [44]. Thus our targets are Types III, IV, V and VI.

Notation Throughout the paper, we let the symbol $\mathcal{X}$ denote one of these types, ie, $\mathcal{X}=$ III, IV, V or VI.

To describe the knots in each Type $\mathcal{X}$, in [11], Berge defined five parameters $\delta, \varepsilon \in\{ \pm 1\}$ and $A, B, b \in \mathbf{Z}$ (satisfying certain conditions depending on $\mathcal{X}$ ). We introduce two new parameters $k, t$ such that $B, b$ are uniquely calculated from $k, t$ and vice-versa. By $K_{\mathcal{X}}(\delta, \varepsilon, A, k, t)$, we mean the knot defined by the parameters in Type $\mathcal{X}$. (Type VI is slightly different from the others.) Taking opposite $\delta$ corresponds to the mirror image of the knot. Note that, if a lens space surgery $(K, r)$ belongs to Type $\mathcal{X},(K !,-r)$ is also a lens space surgery and belongs to the same Type $\mathcal{X}$, where $K$ ! is the mirror image of $K$. See Section 2 for details on the parameters.

The theory of A'Campo's divide knots and links comes from singularity theory of complex curves. The divide is (originally) a relative, generic immersion of a 1-manifold in a unit disk in $\mathbf{R}^{2}$. A'Campo $[1 ; 2 ; 3 ; 4]$ formulated the way to associate to each divide $C$ a link $L(C)$ in $S^{3}$. In the present paper, we regard a PL (piecewise linear) plane curve as a divide by smoothing the corners. The class of divide links properly contains the class of the links arising from isolated singularities of complex curves, ie, positive torus knots and iterated torus knots satisfying certain inequalities in their parameters.

Definition 1.1 Let $X$ be the $\pi / 4$-lattice defined by $\{(x, y) \mid \cos \pi x=\cos \pi y\}$ in the $x y$-plane $\left(\mathbf{R}^{2}\right)$. By an $L$-shaped region, we mean a union of two rectangles sharing a 


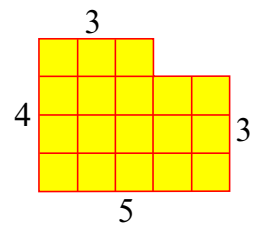

area $=18$
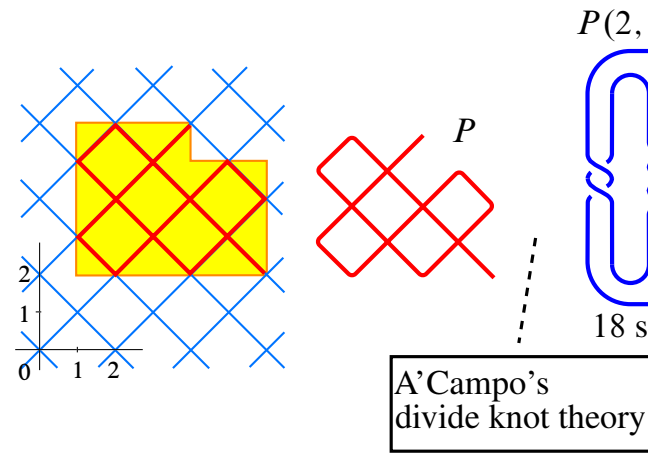

Figure 1: Pretzel knot $(-2,3,7)$ with coefficient 18

corner and overlapping along an edge of one, where the rectangles are assumed to have all edges parallel to either the $x$-axis or $y$-axis, and all vertices at lattice points $\left(\in \mathbf{Z}^{2}\right)$. We call a plane curve an $L$-shaped curve if the curve $P$ is obtained as antersection $X \cap \mathcal{L}$ of $X$ and an $\mathrm{L}-$ shaped region $\mathcal{L}$. We define area $(P)$ of an $\mathrm{L}-$ shaped curve $P=X \cap \mathcal{L}$ as the area (2-dimensional volume) of the $\mathrm{L}$-shaped region $\mathcal{L}$ defining $P$.

See Figure 1. It is the starting example of our results. The L-shaped curve $P=X \cap \mathcal{L}$, as a divide, presents the pretzel knot of type $(-2,3,7)$. Its 18 surgery is a lens space, which is one of the examples of Fintushel and Stern [17]. Note that the area of $P$ is equal to 18 , the coefficient of the lens space surgery. Our main result is:

Theorem 1.2 Up to mirror image, every Berge knot $K$ of lens space surgery in Type $\mathcal{X}$

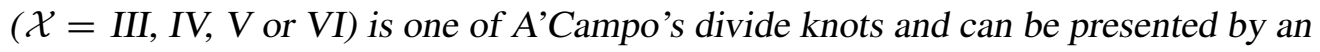
$L$-shaped curve. In fact, one of $K$ or its mirror image is presented by a positive braid (say $w$ ) and the other by a negative one (the inverse $w^{-1}$ ). The divide knot is exactly equal to the positive one, but the choice of $\delta(1$ or -1$)$ depends on the parameters $\mathcal{X}$ and $(\varepsilon, A, k, t)$.

Theorem 1.2 can be proved by combining of Lemma 2.1 and Lemma 3.8. The aim of the present paper is to define the operation adding squares on L-shaped curves and the construction of the $\mathrm{L}$-shaped curves using the operation in Section 4. For the given parameters $\mathcal{X}$ and $(\delta, \varepsilon, A, k, t)$, we will construct an L-shaped curve $P_{\mathcal{X}}(\varepsilon, A, k, t)=X \cap \mathcal{L}_{\mathcal{X}}(\varepsilon, A, k, t)$; see Demonstration 4.8.

Theorem 1.3 The Berge knot $K_{\mathcal{X}}(\delta, \varepsilon, A, k, t)$ in Type $\mathcal{X}(\mathcal{X}=I I I, I V, V$ or VI) is presented by our $L$-shaped curve $P_{\mathcal{X}}(\varepsilon, A, k, t)$ up to mirror image:

$L\left(P_{\mathcal{X}}(\varepsilon, A, k, t)\right)=K_{\mathcal{X}}(1, \varepsilon, A, k, t)$ or its mirror image $K_{\mathcal{X}}(-1, \varepsilon, A, k, t)$.

The choice of $\delta(1$ or -1$)$ depends on $\mathcal{X}$ and $(\varepsilon, A, k, t)$. 
Next, we study the surgery coefficients. By the Cyclic Surgery Theorem of CullerGordon-Luecke-Shalen [14], if a hyperbolic knot $K$ admits a lens space surgery, then the coefficient is integral. By $\operatorname{coef}\left(K_{\mathcal{X}}(\delta, \varepsilon, A, k, t)\right)$, we denote the surgery coefficient of the lens space surgery of the knot as in Type $\mathcal{X}$. Note that there exist some hyperbolic knots that have two coefficients of lens space surgery (such coefficients are proved to be consecutive in [14]) and belong to different types as the pairs with the coefficients. This is the reason why we state "as in Type $\mathcal{X}$ ".

Theorem 1.4 Under the correspondence in Theorem 1.3, the area of the $L$-shaped curve $P$ is equal to (the absolute value of) the coefficient of the lens space surgery of $L(P)$ as in Type $\mathcal{X}$, or is greater by one:

$$
\operatorname{area}(P)-|\operatorname{coef}(L(P))|=0 \text { or } 1 .
$$

This theorem will be proved as Lemma 5.3, in which we will decide the choice ( 0 or 1) by the parameters. We will prove $\operatorname{coef}(L(P))>0$ in Lemma 5.1. Thus we will change $|\operatorname{coef}(L(P))|$ to $\operatorname{coef}(L(P))$ in Lemma 5.3.

Our operation adding squares on L-shaped curves is related to blow-down and to full-twists. The $\mathrm{L}$-shaped plane curve $P_{\mathcal{X}}(\varepsilon, A, k, t)$ is constructed by adding squares twice from $P_{\mathcal{X}}(\varepsilon, A, 0,0)$. This is related to the fact that that $K_{\mathcal{X}}(\delta, \varepsilon, A, k, t)$ is obtained by full-twists "twice" from $K_{\mathcal{X}}(\delta, \varepsilon, A, 0,0)$ via $K_{\mathcal{X}}(\delta, \varepsilon, A, k, 0)$. We will show that some Berge knots are obtained from other Berge knots in other types by some twistings. By adding squares on L-shaped curve presentation of knots, we can search such pairs and check such relations easily; see Section 6.

Here we briefly survey the divide presentation of the other Berge knots (in Type VII and later). All knots are considered up to mirror image. Type VII consists of the knots for which the author [42] first gave L-shaped curve presentations. Type VIII contains some knots that are hard (in the author's opinion) to decide whether they are divide knots, and their divide presentation (if they are). The author has shown that every sporadic knot (in Type IX and later) is a divide knot and has shown a method to construct the divide. But he does not know whether it can be presented by an L-shaped curve or not.

Note that there exists a family of $\mathrm{L}$-shaped divide knots whose area $(P)$-surgeries are not lens spacees [43], but such L-shaped divide knots tend to have exceptional Dehn surgeries, to the author's knowledge [41; 44].

This paper is organized as follows: In the next section, we review Berge knots and their parameters in detail. In Section 3, we review A'Campo's divide knot theory and define L-shaped curves. In Section 4, developing a method adding squares, we construct L-shaped curves (regions) for Berge knots. In Section 5, we will prove Theorem 1.3 
and Lemma 5.3, the precise version of Theorem 1.4. Finally, in Section 6, we study some applications of and advantages to presenting Berge knots as divide knots. We place Tables 1 and 2 which give the parameters and L-shaped regions of Berge knots after the reference list for the reader's convenience.

\section{Berge knots of Types III, IV, V and VI}

We recall Berge's parametrization of knots in Type $\mathcal{X}$. We use his original parameters $\delta, \varepsilon, A, B, b$ and a constant $a(:=0$ or 1$)$ defined in [11], and introduce two new parameters $k$ and $t$.

We start with the following:

(1) $-\delta$ and $\varepsilon$ are signs $(\in\{ \pm 1\})$. The opposite $\delta$ corresponds to the mirror image.

- $A$ is a positive integer, whose range and parity (even or odd) depends on $\mathcal{X}$.

- $k$ runs in $\mathbf{N}_{\geq 0} . B$ is decided by $(\varepsilon, A, k)$ and satisfies $0<2 A \leq B$.

- $b, t \in \mathbf{Z}$. They can be negative.

(2) The parameters $k, t$ are introduced instead of the conditions in [11] written by sentences and by congruences, respectively. For example, instead of " $(B+\varepsilon) / A$ is an odd integer" in [11, Table 3, page 15], we set $B=A(3+2 k)-\varepsilon$. Instead of " $b \equiv-2 \varepsilon \delta A(\bmod B)$ " in [11, Table 3, page 15], we set $b=-\delta \varepsilon(2 A+t B)$. These are the relations between $(B, b)$ and $(k, t)$ in Type III. They are similar in other types, but slightly different; see Table 1(1).

(3) The independent parameters are $(\delta, \varepsilon, A, k, t)$ in Types III, IV and V, but are $(\delta, A, t)$ in Type VI. For convenience, we fix $\varepsilon:=-1, k:=0$ in Type VI and formally regard $(\delta, A, t)$ as $(\delta, \varepsilon, A, k, t)=(\delta,-1, A, 0, t)$.

Notation $\operatorname{By} K_{\mathcal{X}}(\delta, \varepsilon, A, k, t)$, we denote the knot parametrized as $(\delta, \varepsilon, A, k, t)$ in Type $\mathcal{X}$, by Berge in [11].

Now we go into the detail. See Table 1(1), (2) and (3). In Table 1(1), we define $B$ and $b$ (depending on $\mathcal{X}$ ), using a temporary parameter $l$. For fixed $A$ and $\varepsilon$, the possible values of $l$ are in an arithmetic sequence, depending on $\mathcal{X}$. We parametrize the sequence by $k \in \mathbf{N}_{\geq 0}$ as in Table 1(2). In every case, the surgery coefficient is $b B+\delta A$, where $B$ depends on $\varepsilon, A, k$. In Table 1(3), we deform the coefficients into the form including the terms $+k A^{2}+t B^{2}$ (or $+k(2 A)^{2}+t B^{2}$ in Type III). These are related to our method adding squares in Section 4 . Note that, if a knot $K$ with coefficient $r$ belongs to Type $\mathcal{X}$, its mirror image $K$ ! with $-r$ (ie, opposite $\delta$ ) also belongs to the same Type $\mathcal{X}$. 
Using these parameters, in [11], Berge has already given the braid presentations of these knots:

Lemma 2.1 (Berge [11]) Every knot $K_{\mathcal{X}}(\delta, \varepsilon, A, k, t)$ is presented as the closure of the braid $W(B)^{b} W(A+1-a)^{\delta}$ of index $B$, where $W(n)=\sigma_{n-1} \sigma_{n-2} \cdots \sigma_{1}$; see Figure 3.

Definition 2.2 We define an antihomomorphic (ie, $\left.\rho\left(\beta_{1} \beta_{2}\right)=\rho\left(\beta_{2}\right) \rho\left(\beta_{1}\right)\right)$ involution $\pi$-rotation $\rho$ on the braid group of index $n$ by extending $\rho\left(\sigma_{i}\right)=\sigma_{n-i}$; see Figure 2 . By $\beta^{\prime} \stackrel{\rho}{=} \beta$, we mean $\beta^{\prime}=\rho(\beta)$ and equivalently $\rho\left(\beta^{\prime}\right)=\beta$.

Using this opportunity, we set another notation, $\beta^{\prime} \sim \beta$, to mean that the closure of $\beta^{\prime}$ is the same knot or link as that of $\beta$. Note that $\beta^{\prime} \stackrel{\rho}{=} \beta$ up to conjugacy implies $\beta^{\prime} \sim \beta$.
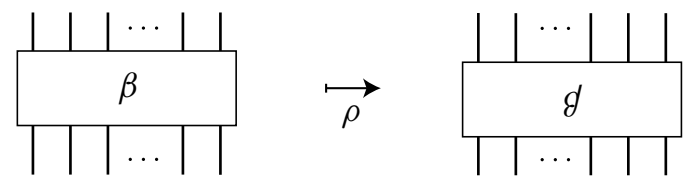

Figure 2: $\pi$-rotation $\rho$

Our L-shaped divide knots are always presented by positive braids (see Section 3), while any divide knot is a closure of a strongly quasi-positive braid (Lemma 3.2(7)). Thus, first, if $b<0$, we take the mirror image (ie, change the sign $\delta$, then $b$ becomes $-b>0)$ and next, we use the following lemma if it is necessary.

Lemma 2.3 Let $a_{1}, a_{2}$ and $c$ be positive integers with $a_{1}<a_{2}$. The closure of the braid $W\left(a_{2}\right)^{c} W\left(a_{1}\right)^{-1}$ of index $a_{2}$ is the same knot as the closure of the braid $W\left(a_{2}\right)^{c-1} W\left(a_{2}-a_{1}+1\right)$.

Proof First, we have

$$
\begin{aligned}
W\left(a_{2}\right) W\left(a_{1}\right)^{-1} & =\sigma_{a_{2}-1} \sigma_{a_{2}-2} \cdots \sigma_{a_{1}} \cdots \sigma_{2} \sigma_{1}\left(\sigma_{a_{1}-1} \cdots \sigma_{2} \sigma_{1}\right)^{-1} \\
& =\sigma_{a_{2}-1} \sigma_{a_{2}-2} \cdots \sigma_{a_{1}} \\
& \stackrel{\rho}{=} W\left(a_{2}-a_{1}+1\right) .
\end{aligned}
$$

Since $\pi$-rotation $\rho$ is antihomomorphic, and the braid $W(n)$ of index $n\left(=a_{2}\right)$ is fixed by $\rho$, we have

$$
\begin{aligned}
W\left(a_{2}\right)^{c} W\left(a_{1}\right)^{-1} & =W\left(a_{2}\right)^{c-1} \cdot W\left(a_{2}\right) W\left(a_{1}\right)^{-1} \\
& \stackrel{\rho}{=} W\left(a_{2}-a_{1}+1\right) W\left(a_{2}\right)^{c-1},
\end{aligned}
$$

which is conjugate to $W\left(a_{2}\right)^{c-1} W\left(a_{2}-a_{1}+1\right)$. 


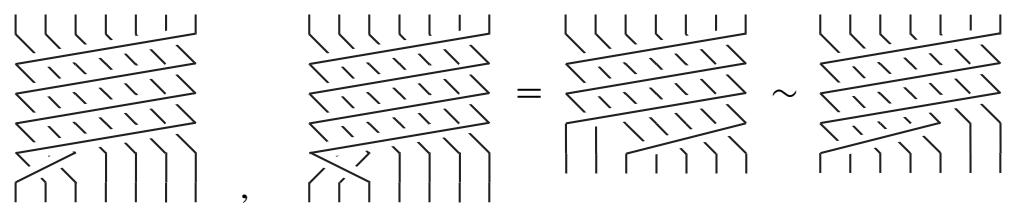

Figure 3: $W(7)^{4} W(3)$ and $W(7)^{4} W(3)^{-1} \sim W(7)^{3} W(5)$

Figure 3 illustrates Lemma 2.3. The knot $K_{\mathrm{III}}(1,-1,2,0,0)$ is presented by the braid $W(7)^{4} W(3)$, and $K_{\mathrm{III}}(-1,1,2,0,0)$ is presented by $W(7)^{4} W(3)^{-1} \sim W(7)^{3} W(5)$.

We end this section with a discussion of the fiberedness of Berge knots. It was one of the motivations of this research, since every divide knot is fibered; see Lemma 3.2(4).

Corollary 2.4 (Teragaito [40], Ni [33]) Every Berge knot of lens space surgery in Types III, IV, V and VI is fibered.

This corollary can be proved by Lemma 2.1, 2.3 and the fact that knots presented by positive (or negative) braids are fibered [38]. In fact, Teragaito [40] (see Hill and Murasugi [27, Section 5.7]), has shown that all Berge knots (including Types VII, ..., XII; see Section 1) are fibered, by proving the braid positivity. See also Baker [7, Appendix B] for Types VII and VIII. Using knot Floer homology, it is proved by Ni [33] that every knot admitting a lens space surgery is fibered rather than just that Berge knots are fibered; see also Hedden [25]. Their argument does not rely upon double primitivity.

\section{L-shaped curves and A'Campo's divide knots}

The theory of A'Campo's divide knots and links $[1 ; 2 ; 3 ; 4]$ comes from singularity theory of complex curves. It is a method to associate to each divide (a plane curve) $C$ a link $L(C)$ in the 3-dimensional sphere $S^{3}$. The original definition of divide knots in [1] is differential-geometric. Hirasawa [28] visualized the construction. We are concerned with the plane curves of special type, called "L-shaped curves"; see Section 3.2. For such special curves, we can use another method introduced by CouturePerron [13]; see Section 3.3.

\subsection{A'Campo's divide knots}

We start with the typical example of divide knots; see Figure 4: 
Lemma 3.1 (Goda-Hirasawa-Y [18]; see also Arnol' d-Guseĭn-Zade-Varchenko [5] and Guseřn-Zade [23]) Let $a, b$ be a pair of positive integers and $\mathcal{R}(a, b)$ be an $a \times b$-rectangle region. A plane curve defined by $X \cap \mathcal{R}(a, b)$ (a billiard curve of type $B(a, b))$ presents the torus link $T(a, b)$ as a divide.
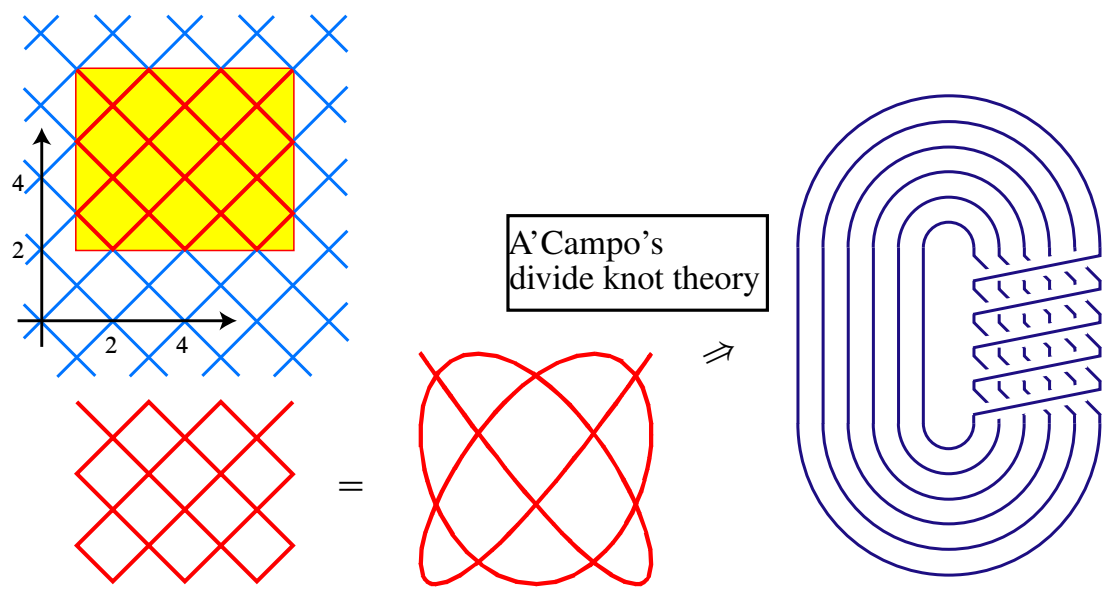

Figure 4: A billiard curve presents a torus knot $(\operatorname{eg} T(6,5))$

Some characterizations of (general) divide knots and links are known, and some topological invariants of $L(P)$ can be gotten from the divide $P$ directly. Here, we list some of them.

Lemma 3.2 ((1)-(6) by A'Campo [2], (7) by Hirasawa [28] and Rudolph [37])

(1) $L(P)$ is a knot (ie, connected) if and if only $P$ is an immersed arc.

(2) If $L(P)$ is a knot, the unknotting number, genus and 4-genus of $L(P)$ are all equal to the number $d(P)$ of the double points of $P$.

(3) If $P=P_{1} \cup P_{2}$ is the image of an immersion of two arcs, then the linking number of the two component link $L(P)=L\left(P_{1}\right) \cup L\left(P_{2}\right)$ is equal to the number of the intersection points between $P_{1}$ and $P_{2}$.

(4) If $P$ is connected, then $L(P)$ is fibered.

(5) A divide $P$ and its mirror image $P$ ! present the same knot or link: $L(P !)=$ $L(P)$.

(6) If $P_{1}$ and $P_{2}$ are related by some $\Delta$-moves, then the links $L\left(P_{1}\right)$ and $L\left(P_{2}\right)$ are isotopic: If $P_{1} \sim_{\Delta} P_{2}$ then $L\left(P_{1}\right)=L\left(P_{2}\right)$; see Figure 5 .

(7) Any divide knot is a closure of a strongly quasi-positive braid, ie, a product of some $\sigma_{i j}$ in Figure 5. 


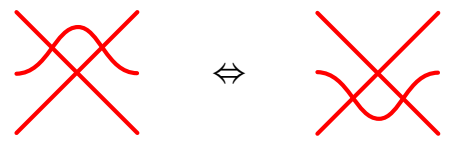

$\Delta$-move

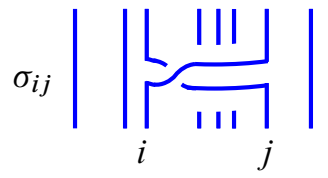

Figure 5: Basics on divide knots

For theory of divide knots, see also Chmutov [12] and Quach Hongler-Weber [36] and "transverse C-links" defined by Rudolph [37]. In [25], Hedden studied some relationship among 4-genus, fibered-ness, (strongly) (quasi-) positivity of braids, and knot Floer homology.

\subsection{Preliminary on L-shaped curves}

First, we parametrize L-shaped regions by four positive integers $a_{1}, a_{2}, b_{1}, b_{2}$ that satisfy $a_{1}<a_{2}$ and $b_{1}<b_{2}$; see Figure 6:

Definition 3.3 (L-shaped region at the origin) In $x y$-plane, we define $L\left[a_{1}, a_{2} ; b_{1}, b_{2}\right]:=\left\{(x, y) \mid 0 \leq x \leq a_{1}, 0 \leq y \leq b_{2}\right\} \cup\left\{(x, y) \mid 0 \leq x \leq a_{2}, 0 \leq y \leq b_{1}\right\}$. By concave corner, we mean the point (of the region) at the coordinate $\left(a_{1}, b_{1}\right)$ in the
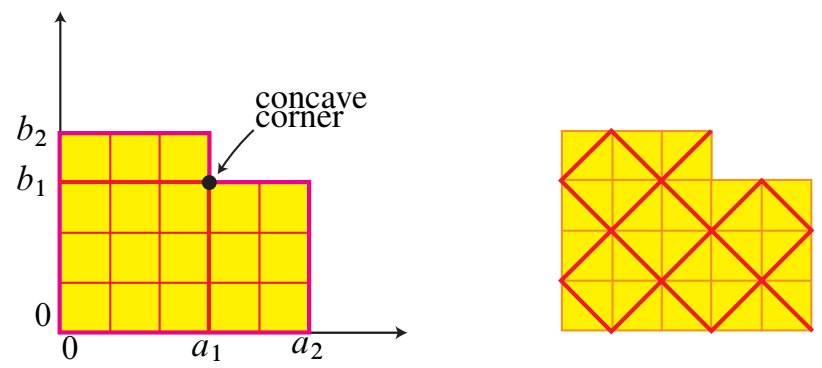

Figure 6: L-shaped region $L[3,5 ; 3,4]$

definition above. We will call not only $L:=L\left[a_{1}, a_{2} ; b_{1}, b_{2}\right]$ but also its transformations $\mathcal{L}=T(L)$ an $L$-shaped region of type $\left[a_{1}, a_{2} ; b_{1}, b_{2}\right]$, where $T$ is a transformation in $x y$-plane generated by the reflection $r_{X}$ along the $x$-axis (Lemma 3.2(5)), the rotation $R$ by $\pi / 2$ about a lattice point and the parallel translation $+\vec{n}$ by a lattice point $\vec{n}\left(\in \mathbf{Z}^{2}\right)$.

Let $X$ be the $\pi / 4$-lattice defined by $\{(x, y) \mid \cos \pi x=\cos \pi y\}$ in $x y$-plane. A lattice point $(m, n)\left(\in \mathbf{Z}^{2}\right)$ is called even (or odd, resp.) if $m+n$ is even (or odd). We 
are concerned only with the case that the intersection $X \cap \mathcal{L}$ is the image of a generic immersed arc. Thus, we always control $+\vec{n}$ and assume:

(*) The concave point of an $\mathrm{L}$-shaped region $\mathcal{L}$ is placed at an odd point.

Assuming (*), the parameter $\left[a_{1}, a_{2} ; b_{1}, b_{2}\right]$ defines a unique plane curve up to isotopy, ie, it depends on neither $r_{X}, R$ nor translations keeping even/odd points. We call the corresponding plane curve $P=X \cap \mathcal{L}$ an $L$-shaped curve of type $\left[a_{1}, a_{2} ; b_{1}, b_{2}\right]$. Of course, for an L-shaped curve $P$ of type $\left[a_{1}, a_{2} ; b_{1}, b_{2}\right]$, we have

$$
\operatorname{area}(P)=a_{2} b_{1}+a_{1} b_{2}-a_{1} b_{1}
$$

On the other hand, the number $d(P)$ of double points of $P$ is

$$
d(P)=\left\{a_{2}\left(b_{1}-1\right)+b_{2}\left(a_{1}-1\right)-a_{1} b_{1}+1\right\} / 2,
$$

because double points are the even points of the interior of the L-shaped region.

The condition $(*)$ is not sufficient for $X \cap \mathcal{L}$ to be the image of an immersed arc. In fact, it possibly consists of multiple components or contains some circle components.

The following proposition follows from Lemma 3.2(2).

Proposition 3.4 If the $L$-shaped curve $P=X \cap \mathcal{L}$ of type $\left[a_{1}, a_{2} ; b_{1}, b_{2}\right]$ with the assumption $(*)$ is an immersed arc, then the genus $g(L(P))$ of the divide knot $L(P)$ is equal to the number $d(P)$ of the double points of $P$ (the unknotting number and the 4-genus are also):

$$
g(L(P))=\left\{a_{2}\left(b_{1}-1\right)+b_{2}\left(a_{1}-1\right)-a_{1} b_{1}+1\right\} / 2 .
$$

Thus, it holds that area $(P)-2 g(L(P))=a_{2}+b_{2}-1$.

\subsection{L-shaped divide knots}

In [13] Couture and Perron pointed out a method to get the braid presentation from the divide (the plane curve) in the restricted cases, called "ordered Morse" divides. Our L-shaped curves are all ordered Morse, thus we can apply their method. It is a special case of Hirasawa's method in [28].

Lemma 3.5 The divide link presented by the L-shaped curve of type $\left[a_{1}, a_{2} ; b_{1}, b_{2}\right]$ is the closure of the braid $W\left(a_{2}\right)^{b_{1}} W\left(a_{1}\right)^{b_{2}-b_{1}}$ of index $a_{2}$, where $W(n)$ is the braid $\sigma_{n-1} \sigma_{n-2} \cdots \sigma_{1}$. 
Such a link should be regarded as a "rationally twisted" torus link in the following sense: The link is obtained by a " $\left(b_{2}-b_{1}\right) / a_{1}$ twist" of the parallel $a_{1}$ strings in $a_{2}$ strings of torus link $T\left(a_{2}, b_{1}\right)$ in the standard position $W\left(a_{2}\right)^{b_{1}}$.

Example 3.6 The divide knot presented by the L-shaped curve $[3,5 ; 3,4]$ is the closure of the braid $\left(\sigma_{2} \sigma_{4} \sigma_{1} \sigma_{3}\right)^{3} \sigma_{2} \sigma_{1}$ (conjugate to $\left.\left(\sigma_{4} \sigma_{3} \sigma_{2} \sigma_{1}\right)^{3} \sigma_{2} \sigma_{1}\right)$ of index 5 , which is $P(-2,3,7)$.

Proof First, we define the words $o(n)$ and $e(n)$ in the braid group of index $a_{2}$ :

$$
e(n):=\prod_{i \text { even }, i<n} \sigma_{i}, \quad o(n):=\prod_{i \text { odd }, i<n} \sigma_{i},
$$

where $n$ is a positive integer less than or equal to the index. Note that $\sigma_{i}$ and $\sigma_{j}$ are commutative if $i$ and $j$ have the same parity. If $j \leq k<l$ and $k$ is even, $o(k)^{-1} o(l)$ is a product of $\sigma_{i}$ 's with $i \geq k+1$, thus is commutative with both $o(j)$ and $e(j)$. Similarly, if $j \leq k<l$ and $k$ is odd, then $e(k)^{-1} e(l)$ is commutative with both $o(j)$ and $e(j)$.

In the case of L-shaped curves, Couture-Perron's method is summarized as the algorithm in Figure 7. By direct application of the algorithm to the L-shaped curve of type,

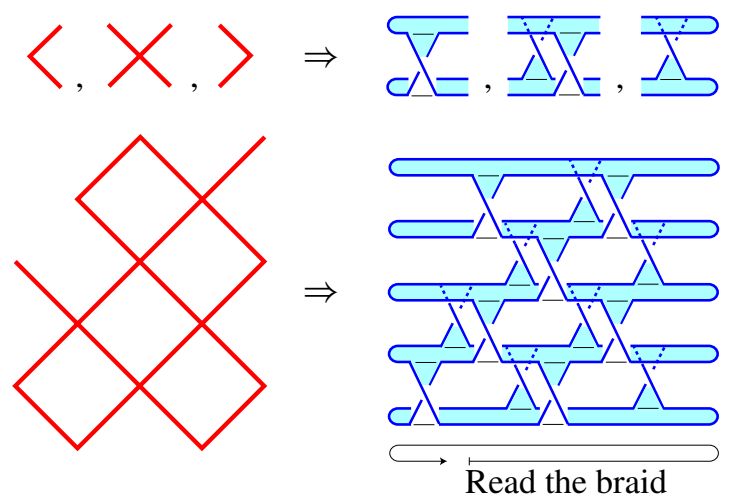

Figure 7: Couture-Perron's method in the case $[3,5 ; 3,4](\pi / 2$ rotated $)$

we have:

Claim 1 [13] The $L$-shaped curve of type $\left[a_{1}, a_{2} ; b_{1}, b_{2}\right]$ presents the closure of the braid

$$
B\left[a_{1}, a_{2} ; b_{1}, b_{2}\right]:= \begin{cases}\left(e\left(a_{2}\right) o\left(a_{2}\right)\right)^{b_{1}}\left(e\left(a_{1}\right) o\left(a_{1}\right)\right)^{b_{2}-b_{1}} & \text { if } a_{1} \text { is odd } \\ \left(o\left(a_{2}\right) e\left(a_{2}\right)\right)^{b_{1}}\left(o\left(a_{1}\right) e\left(a_{1}\right)\right)^{b_{2}-b_{1}} & \text { if } a_{1} \text { is even. }\end{cases}
$$


The key idea of the rest of the proof is in Figure 8.
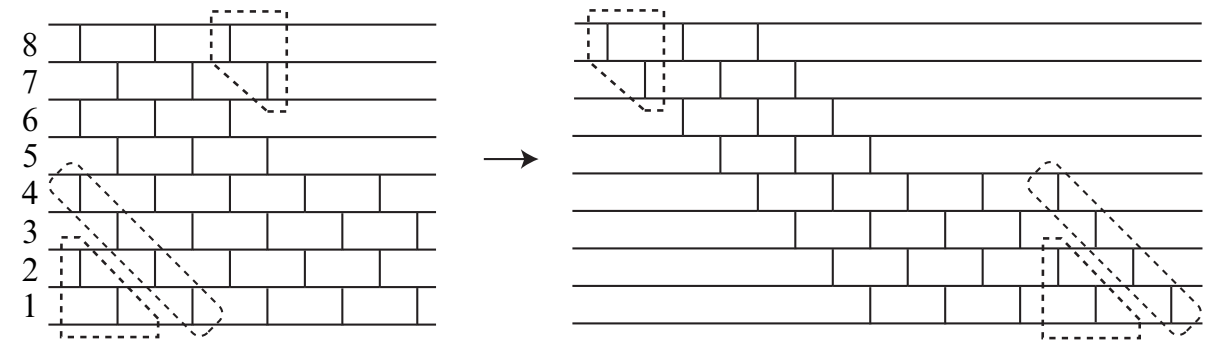

Figure 8: Braid of L-shaped curve $[5,9 ; 3,5]$

Let $G(n)$ be as follows:

$$
G(n):= \begin{cases}e(n+1) o(n) e(n-1) o(n-2) e(n-3) \cdots e(4) o(3) & \text { if } n \text { is odd } \\ o(n+1) e(n) o(n-1) e(n-2) o(n-3) \cdots e(4) o(3) & \text { if } n \text { is even }\end{cases}
$$

It is a product of some $\sigma_{i}$ 's with $i<n$.

Claim 2 The following relationships hold:

$$
\begin{aligned}
& G(n-2)^{-1} e(n) o(n) G(n-2)=W(n) \quad \text { if } n \text { is odd, } \\
& G(n-2)^{-1} o(n) e(n) G(n-2)=W(n) \quad \text { if } n \text { is even. }
\end{aligned}
$$

In fact, if $n$ is odd, $e(n-1)^{-1} e(n)=\sigma_{n-1}$ and it commutes with $o(n-2)^{-1}$. Next, $o(n-2)^{-1} o(n)=\sigma_{n-2}$ and it commutes with $e(n-3)^{-1}$. We repeat such reductions until $o(3)^{-1} o(5)=\sigma_{3}$ inductively, and end with $e(4) o(3)=\sigma_{2} \sigma_{1}$. The other case is proved similarly.

Next, we set $H\left(a_{1}, a_{2}\right):=\rho\left(G\left(a_{2}-a_{1}-1\right)\right)$, where $\rho$ is $\pi$-rotation in Definition 2.2. Then, $H\left(a_{1}, a_{2}\right)$ is a product of some $\sigma_{i}$ 's with $i>a_{1}+1$. Thus we have:

Claim $3 H\left(a_{1}, a_{2}\right)$ commutes with $e\left(a_{1}\right), o\left(a_{1}\right)$ and $G\left(a_{1}-2\right)$.

Let $\Omega\left(a_{1}, a_{2}\right):=H\left(a_{1}, a_{2}\right)^{-1} G\left(a_{1}-2\right)$.

Claim 4 By Claims 2 and 3, the following holds:

$$
\begin{array}{ll}
\Omega\left(a_{1}, a_{2}\right)^{-1} e\left(a_{1}\right) o\left(a_{1}\right) \Omega\left(a_{1}, a_{2}\right)=W\left(a_{1}\right) & \text { if } a_{1} \text { is odd, } \\
\Omega\left(a_{1}, a_{2}\right)^{-1} o\left(a_{1}\right) e\left(a_{1}\right) \Omega\left(a_{1}, a_{2}\right)=W\left(a_{1}\right) & \text { if } a_{1} \text { is even. }
\end{array}
$$


The following is the most troublesome step.

Claim 5 We have the following equalities:

$$
\begin{aligned}
& \Omega\left(a_{1}, a_{2}\right)^{-1} e\left(a_{2}\right) o\left(a_{2}\right) \Omega\left(a_{1}, a_{2}\right)=W\left(a_{1}\right) W\left(a_{2}\right) W\left(a_{1}\right)^{-1} \text { if } a_{1} \text { is odd, } \\
& \Omega\left(a_{1}, a_{2}\right)^{-1} o\left(a_{2}\right) e\left(a_{2}\right) \Omega\left(a_{1}, a_{2}\right)=W\left(a_{1}\right) W\left(a_{2}\right) W\left(a_{1}\right)^{-1} \text { if } a_{1} \text { is even. }
\end{aligned}
$$

To prove Claim 5, we divide the braid of index $a_{2}$ into two parts, lower and higher parts along the $a_{1}$-th string. Here we denote $a_{2}-a_{1}+1$ by $\overline{a_{1}}$. In the case $a_{1}$ is odd,

$$
e\left(a_{2}\right) o\left(a_{2}\right)= \begin{cases}e\left(a_{1}\right) o\left(a_{1}\right) \rho\left(e\left(\overline{a_{1}}\right) o\left(\overline{a_{1}}\right)\right) & \text { if } a_{2} \text { is odd, } \\ e\left(a_{1}\right) o\left(a_{1}\right) \rho\left(o\left(\overline{a_{1}}\right) e\left(\overline{a_{1}}\right)\right) & \text { if } a_{2} \text { is even. }\end{cases}
$$

In the former case, by Claim 3, the conjugation of $e\left(a_{2}\right) o\left(a_{2}\right)$ by $\Omega\left(a_{1}, a_{2}\right)$ is divided as the product of that of $e\left(a_{1}\right) o\left(a_{1}\right)$ by $G\left(a_{1}-2\right)$ and that of $\rho\left(e\left(\overline{a_{1}}\right) o\left(\overline{a_{1}}\right)\right)$ by $H\left(a_{1}, a_{2}\right)^{-1}$. Since $H\left(a_{1}, a_{2}\right)$ is defined as $\rho\left(G\left(\overline{a_{1}}-2\right)\right)$,

$$
\begin{aligned}
H\left(a_{1}, a_{2}\right) \rho\left(e\left(\overline{a_{1}}\right) o\left(\overline{a_{1}}\right)\right) H\left(a_{1}, a_{2}\right)^{-1} & =\rho\left(G\left(\overline{a_{1}}-2\right)^{-1} e\left(\overline{a_{1}}\right) o\left(\overline{a_{1}}\right) G\left(\overline{a_{1}}-2\right)\right) \\
& =\rho\left(W\left(\overline{a_{1}}\right)\right) \\
& =W\left(a_{2}\right) W\left(a_{1}\right)^{-1} .
\end{aligned}
$$

Here, we use Claim 2 with odd $n=\overline{a_{1}}$. In the other cases, including when $a_{2}$ is even, the proofs are similar.

Finally, by Claims 1, 4 and 5,

$$
\begin{aligned}
\Omega\left(a_{1}, a_{2}\right)^{-1} B\left[a_{1}, a_{2} ; b_{1}, b_{2}\right] \Omega\left(a_{1}, a_{2}\right) & =\left(W\left(a_{1}\right) W\left(a_{2}\right) W\left(a_{1}\right)^{-1}\right)^{b_{1}} W\left(a_{1}\right)^{b_{2}-b_{1}} \\
& =W\left(a_{1}\right) W\left(a_{2}\right)^{b_{1}} W\left(a_{1}\right)^{-1} W\left(a_{1}\right)^{b_{2}-b_{1}} .
\end{aligned}
$$

This is conjugate to $W\left(a_{2}\right)^{b_{1}} W\left(a_{1}\right)^{b_{2}-b_{1}}$. The proof of Lemma 3.5 is complete. $\square$

By the symmetry between the L-shaped curve of type $\left[a_{1}, a_{2} ; b_{1}, b_{2}\right]$ and that of type $\left[b_{1}, b_{2} ; a_{1}, a_{2}\right]$, we have an extension of the well-known symmetry $T(b, a)=T(a, b)$ of torus knots.

Corollary 3.7 The closures of the braids

$W\left(b_{2}\right)^{a_{1}} W\left(b_{1}\right)^{a_{2}-a_{1}}$ of index $b_{2}$ and $W\left(a_{2}\right)^{b_{1}} W\left(a_{1}\right)^{b_{2}-b_{1}}$ of index $a_{2}$ define the same link.

Lemma 3.8 Let $a_{1}, a_{2}$ and $c$ be positive integers with $a_{1}<a_{2}$, and $\delta$ be a sign $(\in\{ \pm 1\})$. Then, the knot of the closure of the braid of type $W\left(a_{2}\right)^{ \pm c} W\left(a_{1}\right)^{\delta}$ of index $a_{2}$ is presented as a divide knot presented by an L-shaped curve, up to mirror image: 
$(++)$ The knot $W\left(a_{2}\right)^{c} W\left(a_{1}\right)$ is presented by the $L$-shaped curve $\left[a_{1}, a_{2} ; c, c+1\right]$.

$(+-)$ The knot $W\left(a_{2}\right)^{c} W\left(a_{1}\right)^{-1}$ is presented by the L-shaped curve $\left[a_{2}-a_{1}+\right.$ $\left.1, a_{2} ; c-1, c\right]$.

(--) The knot $W\left(a_{2}\right)^{-c} W\left(a_{1}\right)^{-1}$ is the mirror image of the knot presented by the $L$-shaped curve $\left[a_{1}, a_{2} ; c, c+1\right]$.

(-+) The knot $W\left(a_{2}\right)^{-c} W\left(a_{1}\right)$ is the mirror image of the knot presented by the $L-$ shaped curve $\left[a_{2}-a_{1}+1, a_{2} ; c-1, c\right]$.

Proof The case $(++)$ in the lemma follows from Lemma 3.5 directly, and $(+-)$ follows from Lemma 2.3 and Lemma 3.5. The cases $(--)$ and $(-+)$ follow from $(++)$ and $(+-)$ respectively, since, if a knot $K$ is the closure of the braid $w$, then the mirror image $K$ ! is that of the inverse $w^{-1}$, in general.

Proof of Theorem 1.2 By Lemma 2.1 and Lemma 3.8, Theorem 1.2 is already proved: Up to mirror image, every Berge knot of lens space surgery in Types III, IV, V and VI is one of A'Campo's divide knots, and can be presented by an L-shaped curve.

\section{Construction of L-shaped curves}

We define the operation adding squares on L-shaped curves (via L-shaped regions), its drawing notation, and explain how to construct the L-shaped curves $P_{\mathcal{X}}(\varepsilon, A, k, t)=$ $X \cap \mathcal{L}_{\mathcal{X}}(\varepsilon, A, k, t)$ for Berge knots in Type $\mathcal{X}$, given by the parameters $(\delta, \varepsilon, A, k, t)$. From now on, we consider only L-shaped divide knots, ie, the case that the L-shaped curve $P$ (with the assumption $(*)$ in Section 3.2) is the image of an immersed arc (Lemma 3.2(1)).

\subsection{Adding squares I}

We start with the following:

Definition 4.1 For a positive integer $n$, we call the operation on L-shaped curves, changing from that of type $\left[a_{1}, a_{2} ; b_{1}, b_{2}\right]$ to $\left[a_{1}, a_{2}+n b_{1} ; b_{1}, b_{2}\right]$ or $\left[a_{1}+n b_{2}, a_{2}+\right.$ $\left.n b_{2} ; b_{1}, b_{2}\right]$ adding $n$ squares; see examples in Figure 9. As a drawing notation, we specify the edge along which the squares are added, and write $n$ near the edge. By the symmetry in Corollary 3.7, we also call the changing from $\left[a_{1}, a_{2} ; b_{1}, b_{2}\right]$ to $\left[a_{1}, a_{2} ; b_{1}, b_{2}+n a_{2}\right]$ or $\left[a_{1}, a_{2} ; b_{1}+n a_{2}, b_{2}+n a_{2}\right]$ adding $n$ squares. 

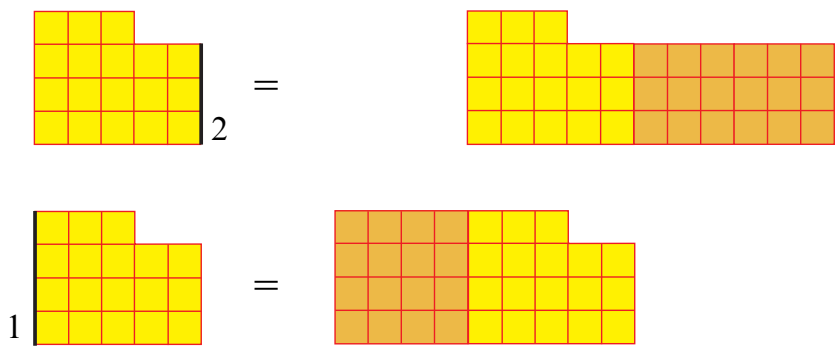

Figure 9: Adding squares I

Lemma 4.2 Adding $n$ squares on an $L$-shaped curve $P$ along an edge (of the region) corresponds to positive (ie, right-handed) $n$ full-twists on the divide knot $L(P)$ along the unknot defined by the edge.

This lemma is proved by using the braid presentation in Lemma 3.5. Note that a full-twist is in the center of the braid group.

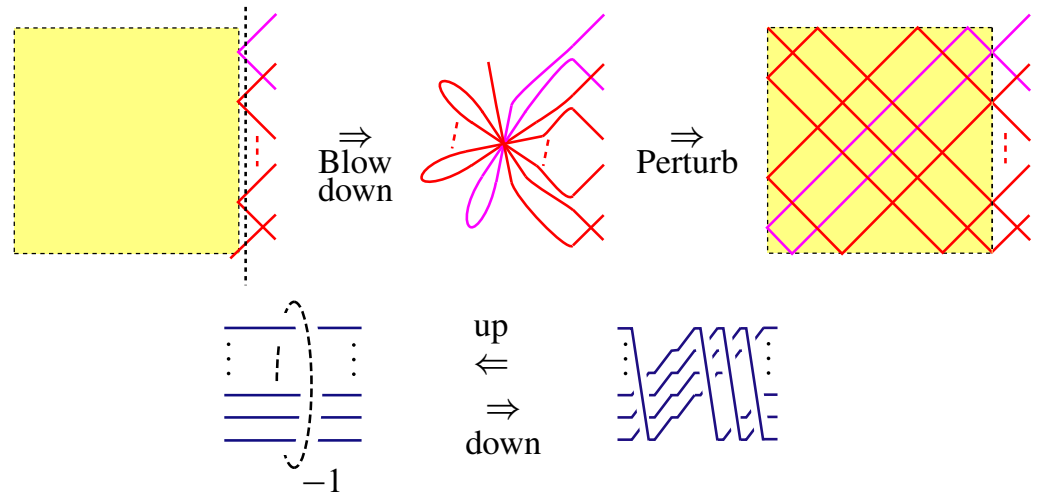

Figure 10: Adding a square corresponds to a full-twist

Adding a square can be regarded as "blow-down", in the following sense. The coordinate transformation $(x, y)=(x, x t)$ (or $=(y t, y))$ is called a blow-up in singularity theory, and is used for resolution of singularity of complex curves; see Harer-Kas-Kirby [24, page 16]. For example, for coprime positive integers $(p, q)$ with $p<q$, the complex curve $x^{q}-y^{p}=0$ becomes $x^{p}\left(x^{q-p}-t^{p}\right)=0$ by the transformation. In this example, the link of the singularity at the origin changes from the torus knot $T(p, q)$ to $T(p, q-p)$ and an unknot defined by the complex line $t$-axis ( $x=0$ in $x t$-plane) appears. The unknot is the axis of the full-twist. It corresponds to the Kirby calculus in the bottom figure in Figure 10, ie, $(-1)$-framed unknot appears and the other 
components change by a left-handed full-twist along the unknot. Framings also change by a certain formula. See Kirby [29] and Gompf-Stipsicz [20] for Kirby calculus.

We call its inverse operation, ie, the transformation $(x, y)=\left(x, y^{\prime} / x\right)$, a blow-down. (For example, $y^{2}=x+\epsilon$ becomes $y^{\prime 2}=x^{2}(x+\epsilon)$, where both hand-sides are multiplied by $x^{2}$.) Let $C$ be a real plane curve in $x y$-plane that intersects with $y$-axis transversely. By the transformation, the all intersection points $C$ and $y$-axis concentrate to the origin of $x y^{\prime}$-plane, and the left half $(x<0)$ of $C$ turns upside down along $x$-axis; see the first arrow in Figure 10.

For adding a square on an L-shaped curve along an edge of length $l$, we first blowdown the curve, where we take $x y$-coordinates such that the $y$-axis is parallel and sufficiently close to the edge. After that, we perturb the curve near the multiple crossing at the origin; see the second arrow in Figure 10. By using some $\Delta$-moves in Lemma 3.2(6), we can move the curve into the required L-shaped curve of the square added L-shaped region. The number of double points increases by $l(l-1) / 2$. For the Kirby calculus, see the bottom figure in Figure 10 again. By the operation, the knot $K$ changes by a right-handed full-twist along the unknot defined by the edge. If $K$ is framed (ie, with a surgery coefficient), the framing increases by the square of the linking number of $K$ and $u$. The linking number is equal to the length $l$ of the edge by Lemma 3.2(3).

\subsection{Adding squares II}

We can apply the operation adding squares twice successively by changing the edges, as in Figure 11. In the same figure, we also define a drawing notation. It is important which edge we apply the operation first.

Here, we state the effect of twice adding squares on the knots in $S^{3}$. This is proved by Lemma 4.2.

Lemma 4.3 Suppose that $P=X \cap \mathcal{L}$ be an $L$-shaped curve and the first edge $e_{1}$ and the second one $e_{2}$ of the region $\mathcal{L}$ are specified. By $P^{\prime \prime}=X \cap \mathcal{L}^{\prime \prime}$, we denote the resulting $L$-shaped curve obtained by adding $n_{1}$ squares along $e_{1}$ first and adding $n_{2}$ squares along $e_{2}$ second, successively. Then, the divide knot $L\left(P^{\prime \prime}\right)$ is equal to the knot $K^{\prime \prime}$ obtained by two twistings from $L(P)$ in $S^{3}$ in the following sense:

First, we take the three component divide link $L\left(P \cup c_{1} \cup c_{2}\right)=K \cup L_{1} \cup L_{2}$ presented by the plane curve $P \cup c_{1} \cup c_{2}$, where $L(P)=K$ and $L_{i}$ is the component presented by slightly pushed off $c_{i}$ of $e_{i}(i=1,2)$ into $\mathcal{L}$; see Figure 12 . Note that $L_{1} \cup L_{2}$ is a Hopf link. Next, we take $n_{1}$ full-twists of $K \cup L_{2}$ along $L_{1}$. We call the resulting link $K^{\prime} \cup L_{2}^{\prime}$. Finally, we take $n_{2}$ full-twists of $K^{\prime}$ along $L_{2}^{\prime}$. We call the resulting knot $K^{\prime \prime}$. 

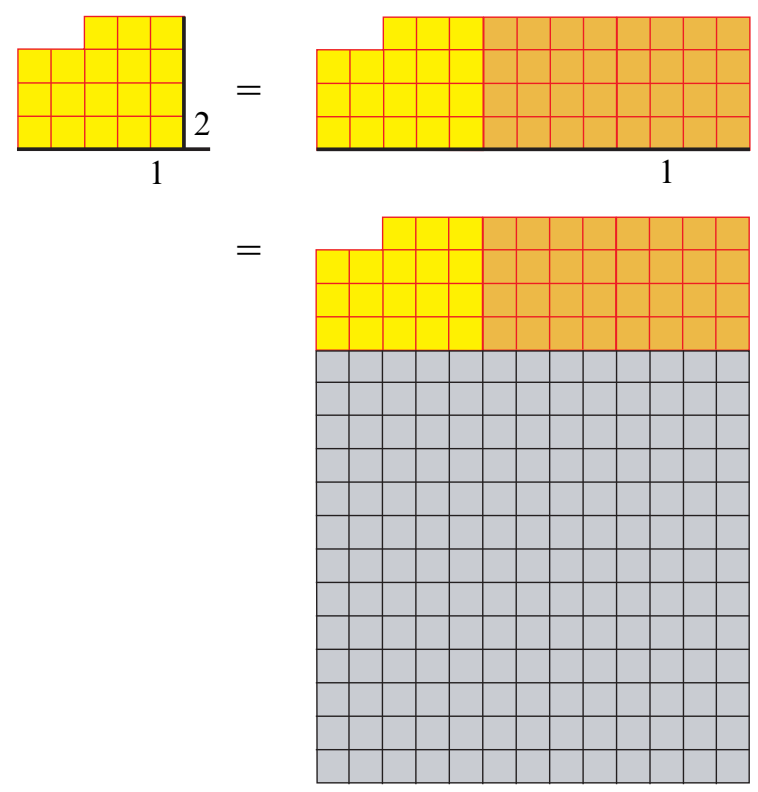

Figure 11: Adding squares II
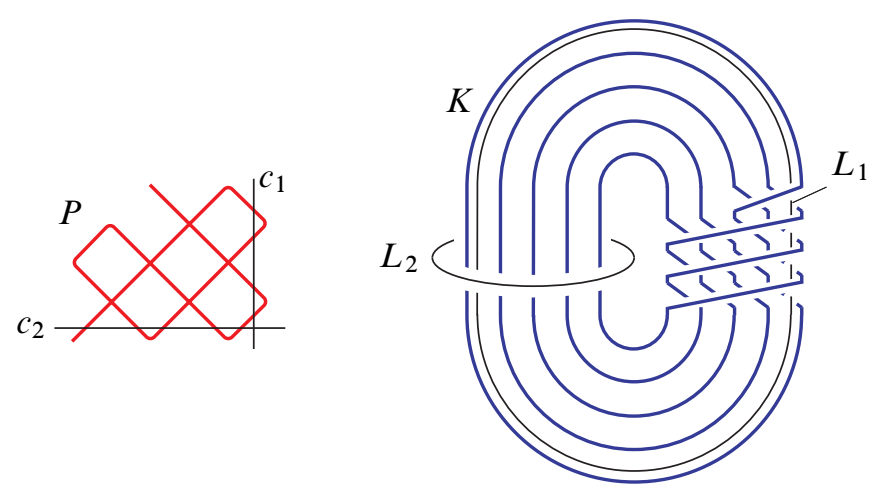

Figure 12: The effect of adding squares twice

In Lemma 4.3, $n_{1}, n_{2}$ are supposed to be positive, however, regarding the statement as a construction of the knot $K^{\prime \prime}$ from $L(P)$ by two twistings, it works also in the case $n_{1}, n_{2}<0$. The knot $K^{\prime \prime}$ may be no longer a divide knot, by the obstruction of braid (quasi-)positivity of divide knots in Lemma 3.2(7) (and Lemma 3.5). In the next subsection, we consider the case $K^{\prime \prime}$ is the mirror image of an L-shaped divide knot. 


\subsection{Adding squares III}

We extend the operation adding $n$ squares into the case $n<0$ partially, in analogy with Lemma 4.3. It corresponds to negative (ie, left-handed) $|n|$ full-twists, and will be called "adding negative squares". We consider the case that the resulting knot of negative full-twists of an L-shaped divide knot is the mirror image of another L-shaped divide knot under some conditions.

Definition 4.4 (Adding negative squares in a certain case) Let $\mathcal{L}$ be an L-shaped region of type $\left[a_{1}, a_{2} ; b_{1}, b_{2}\right]$ with a specified edge $e$. We assume that the edge is the (bottom) one of length $a_{2}$,

$$
b_{2}=b_{1}+1 \quad \text { and } \quad|n| a_{2}>b_{1}+1 .
$$

Only under this condition, we define adding $n$ squares with $n<0$ along $e$ as that the resulting region is of type

$$
\left[a_{2}-a_{1}+1, a_{2} ;|n| a_{2}-b_{1}-1,|n| a_{2}-b_{1}\right] .
$$

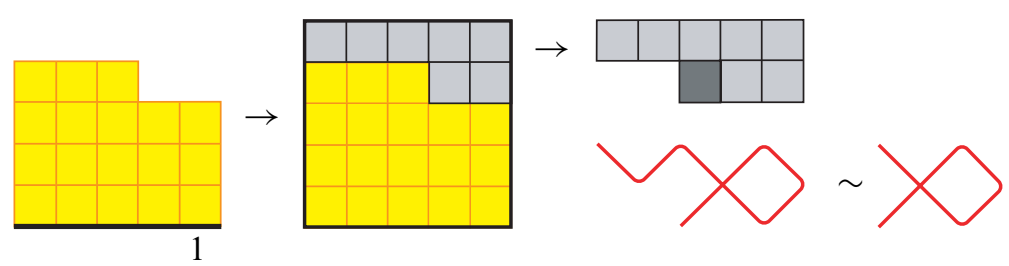

Figure 13: Adding negative squares

This operation should be regarded geometrically as follows; see Figure 13: We assume that the initial L-shaped region is at the origin as in Definition 3.3 (once forgetting the assumption $(*))$ to explain the operation by using $x y$-coordinate. Then adding $n$ squares with $n<0$ is defined as

$$
\begin{aligned}
\operatorname{cl}\left(\left\{(x, y)\left|0 \leq x \leq a_{2}, 0 \leq y \leq\right| n \mid a_{2}\right\} \backslash \mathcal{L}\right) & \\
& \cup\left\{(x, y) \mid a_{1}-1 \leq x \leq a_{1}, b_{1} \leq y \leq b_{1}+1\right\} .
\end{aligned}
$$

By the finally added unit square at the concave point, if the concave point of the initial region is at an odd point, then that of the resulting region is also at an odd point, ie, we can keep the condition $(*)$.

Lemma 4.5 Under the condition of adding negative squares in Definition 4.4, adding $n$ squares with $n<0$ on an $L$-shaped curve $P$ along the edge corresponds to taking the mirror image of $n$ right-handed (ie, $|n|$ left-handed) full-twists on the divide knot $L(P)$ along the unknot defined by the edge. 
Proof By the operation, the type of $\mathrm{L}$-shaped curves is changed from $\left[a_{1}, a_{2} ; b_{1}, b_{1}+1\right]$ to $\left[a_{2}-a_{1}+1, a_{2} ;|n| a_{2}-b_{1}-1,|n| a_{2}-b_{1}\right]$. By Lemma 3.5, the initial curve presents the closure of the braid $W\left(a_{2}\right)^{b_{1}} W\left(a_{1}\right)$ of index $a_{2}$. The edge presents the braid axis in $S^{3}$, The positive full-twist is $W\left(a_{2}\right)^{a_{2}}$ in this situation, and is in the center of the braid group. The resulting knot of the $n$ full-twists is the closure of $W\left(a_{2}\right)^{b_{1}+n a_{2}} W\left(a_{1}\right)=W\left(a_{2}\right)^{-\left(|n| a_{2}-b_{1}\right)} W\left(a_{1}\right)$. Note that $|n| a_{2}>b_{1}+1$ is assumed. Lemma 4.5 follows from Lemma 3.8(-+).

Example 4.6 The example (from $[3,5 ; 3,4]$ to $[3,5 ; 1,2]$ ) in Figure 13 shows that the mirror image of $T(2,3)$ is obtained by $P(-2,3,7)$ (in Example 3.6$)$ by $(-1)$ full-twist along the unknot defined by the bottom edge, whose linking number with $P(-2,3,7)$ is \pm 5 . Note that $\mathrm{L}$-shaped curve of type $[3,5 ; 1,2]$ is the same curve with the billiard curve $B(2,3)$ defined in Lemma 3.1.

Question 4.7 Extend the operation adding negative squares into (more) general cases.

\subsection{How to construct the L-shaped curve}

Preparation is complete. For each Berge knot $K=K_{\mathcal{X}}(\delta, \varepsilon, A, k, t)$ in Type $\mathcal{X}$, we take the $\mathrm{L}$-shaped region $\mathcal{L}=\mathcal{L}(\varepsilon, A, k, t)$ in Table 2, where we used the drawing notation of adding squares. Then, the plane curve $P=X \cap \mathcal{L}$, as a divide, presents the knot $K$ or its mirror image. In fact, each L-shaped region in Table 2 is carefully constructed such that Berge's braid presentation of the knot in Lemma 2.1 agrees with the braid presentation of the region in Lemma 3.8 under the suitable choice of $\delta$. The proof is in the next section.

In Table 2, we draw each L-shaped region in the case of the smallest $A$.

Demonstration $4.8 K_{\mathrm{III}}(\delta, \varepsilon, A, k, t)$ for $(\delta, \varepsilon, A, k, t)=(1,1,2,2,1)$.

By Table $1(1), B=A(3+2 k)-\varepsilon=13, b=-\delta \varepsilon(2 A+t B)=-17$. By Lemma 2.1, it has a braid presentation $W(13)^{-17} W(3)^{1}$. The surgery coefficient is $b B+\delta A=-219$. According to Table 2, the L-shaped region $\mathcal{L}(\varepsilon, A, k, t)$ with $(\varepsilon, A, k, t)=(1,2,2,1)$ is the $\mathrm{L}-$ shaped region of type $[11,13 ; 16,17]$ (the region at the bottom in Figure 11), whose area is 219. By Lemma 3.5, its corresponding plane curve presents the closure of $W(13){ }^{16} W(11)$. By Lemma 2.3, the knot is equal to the closure of $\left.W(13)\right)^{17} W(3)^{-1}$, which is the mirror image of the required knot. 


\section{Proofs of Theorem 1.3 and Theorem 1.4}

Theorem 1.3 is proved by verifying that Berge's braid presentation of the knot (Lemma 2.1) and that of the L-shaped region in Table 2 (Lemma 3.8) agree, under the suitable choice of the sign $\delta$ in each type. In the proof below, we will also decide the choice of $\delta$ (depending on $\mathcal{X}$ and $(\varepsilon, A, k, t))$. We denote the result by $\delta \mathcal{X}(\varepsilon, A, k, t)$, or $\delta \mathcal{X}$ for short. It will be used in the proof of Lemma 5.1

Proof of Theorem 1.3 Here, we prove Theorem 1.3 only in the case of Type III. The proofs in the other cases are similar.

In Type III, in Table 1(1) and (2), we find

$$
a=0, \quad B=A l-\varepsilon, \quad l=3+2 k, \quad b=-\delta \varepsilon(2 A+t B) .
$$

Thus, Berge's braid presentation $W(B)^{b} W(A+1-a)^{\delta}$ of the knot $K_{\mathrm{III}}(\delta, \varepsilon, A, k, t)$ in Lemma 2.1 by [11] is

$$
W((3+2 k) A-\varepsilon)^{-\delta \varepsilon(2 A+t B)} W(A+1)^{\delta} .
$$

First, we consider the case $k=t=0$.

Case $1+$ (Type III, $\varepsilon=1, k=t=0$ ) In Table 2, we find that the $\mathrm{L}-$ shaped region is of type [ $2 A-1,3 A-1 ; 2 A-1,2 A]$. On the other hand, Berge's presentation (5-1) is now $W(3 A-1)^{-2 \delta A} W(A+1)^{\delta}$. Here we choose $\delta=-1$. We use Lemma 3.8(+-) on the knot of $W(3 A-1)^{2 A} W(A+1)^{-1}$.

Case 1 - (Type III, $\varepsilon=-1, k=t=0$ ) In Table 2, we find that the $\mathrm{L}-$ shaped region is of type $[A+1,3 A+1 ; 2 A, 2 A+1]$. On the other hand, Berge's presentation (5-1) is now $W(3 A+1)^{2 \delta A} W(A+1)^{\delta}$. We choose $\delta=1$. We use Lemma 3.8( ++$)$.

Next, we consider the case $k>0(t=0)$. In this case, we use the symmetry of Corollary 3.7 to verify that the parameter $k$ contributes as the $k$ full-twists on the knots.

Case 2+ (Type III, $\varepsilon=1, k>0, t=0$ ) The L-shaped region in Table 2 is of type $[2 A-1+2 k A, 3 A-1+2 k A ; 2 A-1,2 A]$, which presents the closure of $W(3 A-1+2 k A)^{2 A-1} W(2 A-1+2 k A)$ by Lemma 3.5 . By the symmetry of Corollary 3.7 , it presents the same knot of

$$
W(2 A)^{2 A-1+2 k A} W(2 A-1)^{A}=\left(W(2 A)^{2 A}\right)^{k} W(2 A)^{2 A-1} W(2 A-1)^{A} .
$$

Its first part $\left(W(2 A)^{2 A}\right)^{k}$ means $k$ full-twists. On the other hand, Berge's presentation (5-1) is now $W(3 A-1+2 k A)^{-2 \delta A} W(A+1)^{\delta}$ We choose $\delta=-1$. We use Lemma $3.8(+-)$. 
Case 2- (Type III, $\varepsilon=-1, k>0, t=0$ ) The L-shaped region in Table 2 is of type $[A+1,3 A+1+2 k A ; 2 A, 2 A+1]$, which, by Lemma 3.5, presents the closure of $W(3 A+1+2 k A)^{2 A} W(A+1)$. By Corollary 3.7, it presents the same knot of

$$
W(2 A+1)^{A+1} W(2 A)^{A+2 k A}=W(2 A+1)^{A+1} W(2 A)^{A}\left(W(2 A)^{2 A}\right)^{k} .
$$

Its final part $\left(W(2 A)^{2 A}\right)^{k}$ means $k$ full-twists of $2 A$ strings of the braid of index $2 A+1$. On the other hand, Berge's presentation (5-1) is now $W(3 A+1+2 k A)^{2 \delta A} W(A+1)^{\delta}$ by $(5-1)$. We choose $\delta=1$. We use Lemma $3 \cdot 8(++)$.

Before we go into the case $t \neq 0$, we note the following.

(1) The parameter $t$ can be negative.

(2) The parameters $A, k$ and $B$ are independent from $t$.

(3) The parameter $B$ is equal to the length of the edge that is added $t$ squares.

(4) In every case in Table 2, we can apply the operation adding $t$ squares along the edge, ie, the condition " $b_{2}=b_{1}+1$ and $|t| a_{2}>b_{1}+1$ " for adding negative squares are satisfied, even if $t=-1$.

From now on, we use Berge's [11] braid presentation of $K_{\mathrm{III}}(\delta, \varepsilon, A, k, t)$ in Lemma 2.1 in the form

$$
W(B)^{-\delta \varepsilon(2 A+t B)} W(A+1)^{\delta} .
$$

Case 3+ (Type III, $\varepsilon=1, t>0$ ) The L-shaped region in Table 2 is of type $[B-A, B ; 2 A-1+t B, 2 A+t B]$ with $B=3 A-1+2 k A$. On the other hand, Berge's presentation (5-2) is now $W(B)^{-\delta(2 A+t B)} W(A+1)^{\delta}$ We choose $\delta=-1$. We use Lemma 3.8(+-).

Case 3- (Type III, $\varepsilon=-1, t>0$ ) The L-shaped region in Table 2 is of type $[A+1, B ; 2 A+t B, 2 A+1+t B]$ with $B=3 A+1+2 k A$. On the other hand, Berge's presentation (5-2) is now $W(B)^{\delta(2 A+t B)} W(A+1)^{\delta}$. We choose $\delta=1$. We use Lemma 3.8(++).

Before we consider the case $t<0$, we remark the condition $2 A \leq B$ in Section 2. In fact, $2 A=B$ never occurs.

Case 4+ (Type III, $\varepsilon=1, t<0$ ) We use adding negative squares.

The type of $\mathrm{L}$-shaped region in Table 2 is $[A+1, B ;|t| B-2 A,|t| B-2 A+1]$ with $B=3 A-1+2 k A$. On the other hand, Berge's presentation (5-2) is now $W(B)^{-\delta(2 A+t B)} W(A+1)^{\delta}$. We choose $\delta=1$. We use Lemma $3.8(++)$ on the braid $W(B)^{|t| B-2 A} W(A+1)$. 
Case 4- (Type III, $\varepsilon=-1, t<0)$ We use adding negative squares.

The type of $\mathrm{L}-$ shaped region in Table 2 is $[B-A, B ;|t| B-2 A-1,|t| B-2 A]$ with $B=3 A+1+2 k A$, which presents the closure of $W(B)^{|t| B-2 A-1} W(B-A)$. On the other hand, Berge's presentation (5-2) is now $W(B)^{\delta(2 A+t B)} W(A+1)^{\delta}$. We choose $\delta=-1$. We use Lemma $3.8(+-)$ on the knot of $W(B)^{|t| B-2 A} W(A+1)^{-1}$.

After all, for given parameters $(\varepsilon, A, k, t)$, we have shown that the L-shaped curve $P=$ $X \cap \mathcal{L}$ of the L-shaped region $\mathcal{L}=\mathcal{L}_{\mathrm{III}}(\varepsilon, A, k, t)$ in Table 2 presents $K_{\mathrm{III}}(\delta, \varepsilon, A, k, t)$ for suitable choice of $\delta$.

The proof of Theorem 1.3 in the case of Type III is complete. The cases of the other types are proved by the same argument.

In the proof of Theorem $1.3, \delta_{\text {III }}(\varepsilon, A, k, t)$, the suitable choice of $\delta$ in Type III is determined. Considering the other types, it extends as

$$
\delta_{\mathcal{X}}(\varepsilon, A, k, t)= \begin{cases}-1 & \text { if } \varepsilon \cdot \operatorname{sgn}(t)=+1 \\ 1 & \text { if } \varepsilon \cdot \operatorname{sgn}(t)=-1\end{cases}
$$

where $\operatorname{sgn}(t)=1$ if $t>0$ or $t=0, \operatorname{sgn}(t)=-1$ otherwise. This means simply

$$
-\delta_{\mathcal{X}} \cdot \varepsilon \cdot \operatorname{sgn}(t)=+1
$$

Using $\delta_{\mathcal{X}}$, Theorem 1.3 means simply

$$
L\left(P_{\mathcal{X}}(\varepsilon, A, k, t)\right)=K_{\mathcal{X}}\left(\delta_{\mathcal{X}}, \varepsilon, A, k, t\right) .
$$

Now, we consider the coefficients of the lens space surgeries. Before we start the proof of Theorem 1.4, we show:

Lemma 5.1 The lens space surgery coefficient of $L\left(P_{\mathcal{X}}(\varepsilon, A, k, t)\right)$ is positive.

Proof As in the proof of Theorem 1.3, here we prove the lemma only in the case of Type III. In Table 1(3), we find that the coefficient of $L\left(P_{\mathrm{III}}(\varepsilon, A, k, t)\right)=$ $K_{\mathrm{III}}\left(\delta_{\mathcal{X}}, \varepsilon, A, k, t\right)$ (as in Type III) is

$$
-\delta \mathcal{X} \cdot \varepsilon \cdot\left(6 A^{2}-3 \varepsilon A+k(2 A)^{2}+t B^{2}\right) .
$$

Since $|B|=(3+2 k) A-\varepsilon$ (the length of the longest edge), it holds that $\operatorname{sgn}\left(6 A^{2}-3 \varepsilon A+\right.$ $\left.k(2 A)^{2}+t B^{2}\right)=\operatorname{sgn}(t)$. Thus the sign of the coefficient is equal to $-\delta \mathcal{X} \cdot \varepsilon \cdot \operatorname{sgn}(t)=+1$ by (5-3). The proof of the other types are similar. 
Question 5.2 More generally, if a knot $K$ is a closure of a positive braid and admits a lens space surgery, is the coefficient positive?

Now, we prove a precise version of Theorem 1.4 on the difference between the area $\operatorname{area}(P)$ of the L-shaped curve and the surgery coefficient $\operatorname{coef}(L(P))(>0)$ of the lens space surgery as in Type $\mathcal{X}$.

Lemma 5.3 Under the correspondence in Theorem 1.3,

$$
\operatorname{area}(P)-\operatorname{coef}(L(P))= \begin{cases}0 & \text { if }(-1)^{a} \cdot \varepsilon \cdot \operatorname{sgn}(t)=+1 \\ 1 & \text { if }(-1)^{a} \cdot \varepsilon \cdot \operatorname{sgn}(t)=-1\end{cases}
$$

where $\operatorname{sgn}(t)=1$ if $t>0$ or $t=0, \operatorname{sgn}(t)=-1$ otherwise. See Table 1(1) for the definition of $a(=0$ or 1$)$.

Proof First, in the case $k=t=0$, it is easy to verify the equation in Table 2. The parameters $k$ and $t$ with $t>0$ contribute as adding positive squares. In the operation adding a positive square along an edge of length $x$, the area increases by $x^{2}$. It is compatible with the terms $+k A^{2}+t B^{2}$ (or $+k(2 A)^{2}+t B^{2}$ ) in the surgery coefficients in Table 1(3). In the case $t<0$, we do the operation adding negative squares. Suppose that we get the curve $P_{\text {new }}$ from $P_{\text {old }}$ by adding $t$ squares with $t<0$ along an edge of length $x$. Then

$$
\operatorname{coef}\left(L\left(P_{\text {new }}\right)\right)=-\left(\operatorname{coef}\left(L\left(P_{\text {old }}\right)\right)-|t| x^{2}\right),
$$

since the new divide knot $L\left(P_{\text {new }}\right)$ is the mirror image of the knot obtained by lefthanded $|t|$ twists from $L\left(P_{\text {old }}\right)$. On the other hand,

$$
\operatorname{area}\left(P_{\text {new }}\right)=|t| x^{2}-\operatorname{area}\left(P_{\text {old }}\right)+1,
$$

where the last +1 corresponds to the finally added unit square. Thus

$$
\operatorname{area}\left(P_{\text {new }}\right)-\operatorname{coef}\left(L\left(P_{\text {new }}\right)\right)=1-\left(\operatorname{area}\left(P_{\text {old }}\right)-\operatorname{coef}\left(L\left(P_{\text {old }}\right)\right)\right) \text {. }
$$

We have the lemma.

Note that Berge's constant $a$ in [11, Table 3, page 15] was defined geometrically in the context of doubly primitive knots. It might be curious that $a$ is related to the difference between the area and the coefficient as above.

Goda and Teragaito [19] conjectured an inequality

$$
2 g(K)+8 \leq|r| \leq 4 g(K)-1,
$$


on the surgery coefficient $r$ and the genus $g(K)$ of the hyperbolic lens space surgery $(K, r)$. It is called the "Goda-Teragaito conjecture". By Proposition 3.4 and Lemma 5.3, we have:

Corollary 5.4 Let $\left[a_{1}, a_{2} ; b_{1}, b_{2}\right]$ be the type of the $L$-shaped curve of our presentation (in Table 2) of a Berge knot in Types III, IV, V and VI as a divide knot $L(P)$. Then, the following holds:

$$
\begin{aligned}
& \operatorname{coef}(L(P))-2 g(L(P))=a_{2}+b_{2}-1, \text { or } a_{2}+b_{2}-2, \\
& 4 g(L(P))-\operatorname{coef}(L(P))=\left\{\begin{array}{l}
\operatorname{area}(P)-2\left(a_{2}+b_{2}\right)+2, \\
\operatorname{area}(P)-2\left(a_{2}+b_{2}\right)+3 .
\end{array}\right.
\end{aligned}
$$

\section{Further observation}

Divide presentation of L-shaped divide knots helps us to study the constructions of the knots, and the relationship among the lens space surgeries.

\subsection{Twisted torus knots}

Following Dean [15], by a twisted torus knot $T(p, q ; r, s)$, if $r<p$, we mean the knot obtained from the torus knot $T(p, q)$ by $s$ full-twists of $r$ strings in the $p$ parallel strings of $T(p, q)$ in the standard position. On the other hand, if $r>p$, we mean the knot obtained from the torus knot $T(p, q)$ as a closure of the braid $w(p, q) \sigma_{p} \sigma_{p+1} \cdots \sigma_{r-1}$ by $s$ full-twists of all $r$ strings, where $w(p, q) \sigma_{p} \sigma_{p+1} \cdots \sigma_{r-1}$ is a positive Markov stabilization of the standard braid $w(p, q)$ of $T(p, q)$ of index $p$ to a braid of index $r$. The following lemma follows from the braid presentation in Lemma 3.5 (and Lemma 4.2).

Lemma 6.1 Let $(p, q)$ be a coprime pair of positive integers, and $r, s$ integers satisfying $0<r \neq p$ and $s>0$. The twisted torus knot $T(p, q ; r, s)$ is one of A'Campo's divide knots, and can be presented by an L-shaped curve of type

$$
\begin{cases}{[q, q+r s ; r, p]} & \text { if } r<p, \\ {[r s+1, q+r s ; p, r]} & \text { if } r>p,\end{cases}
$$

as A'Campo's divide knots; see Figure 14.

Note that, a twisted torus knot $T(p, q ; r, s)$ can be accidentally nonhyperbolic, a torus knot $T\left(p^{\prime}, q^{\prime}\right)$, or a cable knot $C\left(T\left(p^{\prime}, q^{\prime}\right) ; m^{\prime}, n^{\prime}\right)$ of a torus knot; see Yamada [44] and Morimoto-Yamada [31] for such phenomena. 

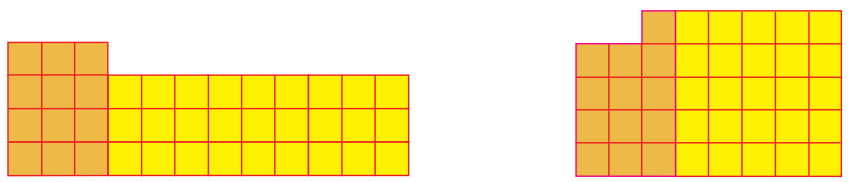

Figure 14: Twisted torus knots $T(p, q ; r, s)(\operatorname{eg} T(4,3 ; 3,3)$ and $T(4,3 ; 5,1))$

Lemma 6.2 Each knot in the following list is a twisted torus knot:

- (Knots with $t=0$ )

$$
\begin{aligned}
K_{\mathrm{III}}(1,-1, A, k, 0) & =T(2 A+1, A+1 ; 2 A, k+1), \\
K_{\mathrm{IV}}(-1,1, A, k, 0) & =T(A, k A+(3 A+1) / 2 ; A-1,1), \\
K_{\mathrm{V}}(-1,1, A, k, 0) & =T(A,(k+1) A+2 ; A-1,1), \\
K_{\mathrm{VI}}(1,-1, A, 0,0) & =T(A-1, A+1 ; A, 1),
\end{aligned}
$$

- (Knots with $t=-1$ )

$$
\begin{aligned}
K_{\mathrm{III}}(1,1, A, k,-1) & =T(A, A+1 ; A-1, k+2), \\
K_{\mathrm{IV}}(-1,-1, A, k,-1) & =T((3 A-1) / 2, A ;(3 A+1) / 2, k+1), \\
K_{\mathrm{V}}(-1,-1, A, k,-1) & =T(2 A-2, A ; 2 A-1, k+1) .
\end{aligned}
$$

Proof The only thing we have to do is to verify them by comparing types of the L-shaped regions, thus we omit the proof in detail. See Figure 15 for the case of $K_{\mathrm{V}}(-1,-1, A, k,-1)$, which needs adding $t$ square with $t=-1$.

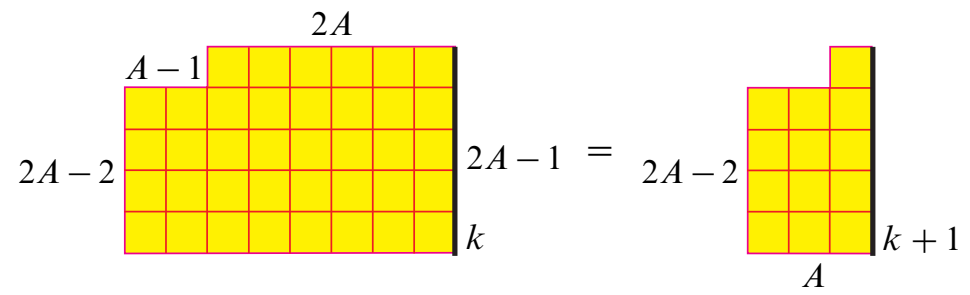

Figure 15: $K_{\mathrm{V}}(-1,-1, A, k,-1)=T(2 A-2, A ; 2 A-1, k+1)$

Now, we count $s$ full-twists along a fixed unknot as one twisting. If after $s_{1}$ full-twists along an unknot we take another $s_{2}$ full-twists along a different unknot, then we count the operation as two twistings. 
Corollary 6.3 [16] Every knot in Berge's Types III, IV, V, and VI is obtained from a twisted torus knot $T_{w}:=T(p, q ; r, s)$ by at most one twisting of $s^{\prime}$ full-twists, thus is obtained from a torus knot at most two successive twistings, such that every knot in the twisting process $\left(T(p, q ; r, i)\right.$ with $0 \leq i \leq s$ and $j$ full-twists of $T_{w}$ with $\left.0 \leq j \leq s^{\prime}\right)$ admits lens space surgery. Furthermore, as a twisted torus knot above, we can take $T(p, q ; r, s)$ that satisfies $|r-p|=1$.

In the process of preparation of this paper, the author was informed by Motegi (see Deruelle-Miyazaki-Motegi [16]) that every knot in Berge's list (including Types VII, ..., XII, see Section 1) is obtained by at most two twistings from a torus knot, which includes the above Corollary.

\subsection{Relations between different types}

See the L-shaped region in the left top figure in Figure 16. It is of type $[3,5 ; 3,4]$, and presents $K_{\mathrm{III}}(-1,1,2,0,0)(=P(-2,3,7))$. The L-shaped regions obtained by adding $k$ squares along the right edge (denoted by $\mathrm{R}$ ), we have a subsequence $K_{\text {III }}(-1,1,2, k, 0)$ in Type III; see Type III $(\varepsilon=1)$ in Table 2 . On the other hand,

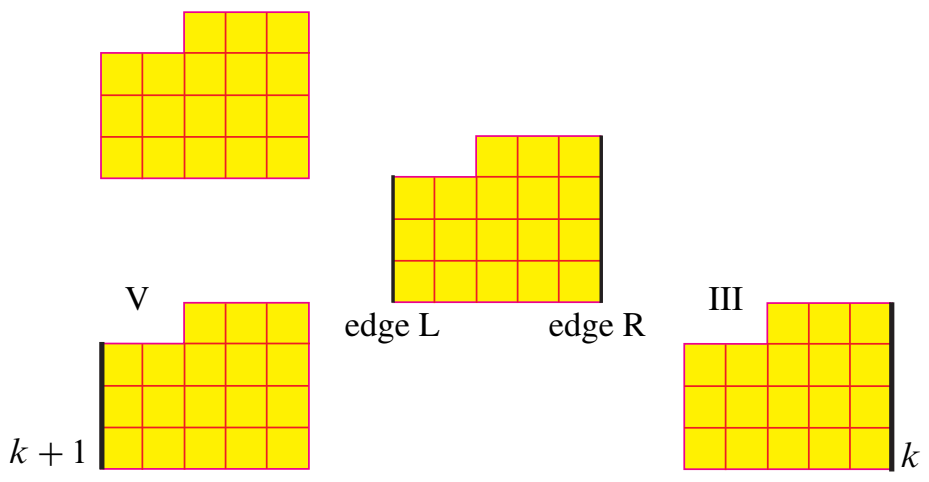

Figure 16: Type III and Type V

those obtained by adding $k+1$ squares with $k \geq 0$ along the left edge (denoted by L), we have another subsequence $K_{\mathrm{V}}(1,1,3, k, 0)$ in Type $\mathrm{V}$ (a different Type); see Type V $(\varepsilon=-1)$ in Table 2. By

$$
K_{\mathrm{III}}(-1,1,2,0,0) \mapsto K_{\mathrm{V}}(1,1,3, k, 0),
$$

we denote such a relation, regarding it as $K_{\mathrm{III}}(-1,1,2,0,0)=$ " $K_{\mathrm{V}}(1,1,3,-1,0)$ ”. Using L-shaped curve presentation in Table 2, we can see more such relations:

- For each $A$ with $A \geq 3, K_{\mathrm{III}}(-1,1, A, 0,0) \mapsto K_{\mathrm{IV}}(1,-1,2 A-1, k, 0)$.

- For each $A, K_{\mathrm{III}}(1,-1, A, 0,0) \mapsto K_{\mathrm{IV}}(-1,1,2 A+1, k, 0)$. 
Question 6.4 Find more such relations, especially in the case $t \neq 0$.

\subsection{Parameters translation}

Parameters of Berge knots are different among some papers. In Table 1(4), we give the translation formula between

$$
(A, k) \text { in }[11] \text { and this paper } \quad \text { and } \quad(n, p) \text { in }[10 ; 7 ; 9 ; 16] .
$$

The signs $\delta, \varepsilon$ are commonly used among these papers.

For example, $K_{\mathrm{IV}}(\delta,-1,5,1, t)$ (ie, $\left.(\varepsilon, A, k)=(-1,5,1)\right)$ in this paper is, up to mirror image, obtained from the knot of $(\varepsilon, n, p)=(-1,2,4)$ in Type IV in $[10 ; 7 ; 9 ; 16]$ by $\pm t$ full-twists.

The parameter $p$ is defined as a positive integer, and our $k$ is just a parallel shift of $p$ such that " $k=0$ at the minimal possible value as $p$ ": the statement " $\varepsilon p \neq-2,-1,0,1$ " (see Table 1(4)) is referred as

$$
\text { " } p \geq 2 \text { if } \varepsilon=+1 \text { and } p \geq 3 \text { if } \varepsilon=-1 " \quad \text { in }[10 ; 7 ; 9 ; 16]
$$

On the other hand, we say

$$
\text { " } k:=p-2 \text { if } \varepsilon=+1 \text { and } k:=p-3 \text { if } \varepsilon=-1 " \text { in the present paper. }
$$

Acknowledgements The author would like to thank to Professor Mikami Hirasawa, Professor Tomomi Kawamura, Professor Masaharu Ishikawa, Professor Sergei Chmutov and Professor Norbert A'Campo for informing him of A'Campo's divide knot theory. The author also would like to thank to Professor Kimihiko Motegi, Professor Masakazu Teragaito, Professor Hiroshi Goda, Professor Noriko Maruyama, Dr Toshio Saito, Dr Kenneth L Baker, Dr Arnaud Deruelle, Dr Hiroshi Matsuda and Professor John Berge for helpful suggestions on lens space surgery. The author would like to express his sincere gratitude to the referee for carefully reading the manuscript and giving him much advice.

This work was supported by KAKENHI (Grant-in-Aid for Scientific Research) No. 18740029. 


\section{References}

[1] N A'Campo, Le groupe de monodromie du déploiement des singularités isolées de courbes planes. I, Math. Ann. 213 (1975) 1-32 MR0377108

[2] N A'Campo, Generic immersions of curves, knots, monodromy and Gordian number, Inst. Hautes Études Sci. Publ. Math. (1998) 151-169 (1999) MR1733329

[3] N A'Campo, Planar trees, slalom curves and hyperbolic knots, Inst. Hautes Études Sci. Publ. Math. (1998) 171-180 (1999) MR1733330

[4] N A'Campo, Real deformations and complex topology of plane curve singularities, Ann. Fac. Sci. Toulouse Math. (6) 8 (1999) 5-23 MR1721511

[5] V I Arnol' d, S M Gusein-Zade, A N Varchenko, Singularities of differentiable maps. Vol. II. Monodromy and asymptotics of integrals, Monogr. in Math. 83, Birkhäuser, Boston (1988) MR966191 Translated from the Russian by H Porteous. Translation revised by the authors and $\mathrm{J}$ Montaldi

[6] J Bailey, D Rolfsen, An unexpected surgery construction of a lens space, Pacific J. Math. 71 (1977) 295-298 MR0488061

[7] K L Baker, Knots on once-punctured torus fibers, PhD thesis, University of Texas at Austin (2004)

[8] K L Baker, Surgery descriptions and volumes of Berge knots. I. Large volume Berge knots, J. Knot Theory Ramifications 17 (2008) 1077-1097 MR2457837

[9] K L Baker, Surgery descriptions and volumes of Berge knots. II. Descriptions on the minimally twisted five chain link, J. Knot Theory Ramifications 17 (2008) 1099-1120 MR2457838

[10] J Berge, Some knots with surgeries yielding lens spaces, Unpublished manuscript (1990)

[11] J Berge, The knots in $D^{2} \times S^{1}$ which have nontrivial Dehn surgeries that yield $D^{2} \times S^{1}$, Topology Appl. 38 (1991) 1-19 MR1093862

[12] S Chmutov, Diagrams of divide links, Proc. Amer. Math. Soc. 131 (2003) 1623-1627 MR1950295

[13] O Couture, B Perron, Representative braids for links associated to plane immersed curves, J. Knot Theory Ramifications 9 (2000) 1-30 MR1749500

[14] M Culler, C M Gordon, J Luecke, P B Shalen, Dehn surgery on knots, Ann. of Math. (2) 125 (1987) 237-300 MR881270

[15] J Dean, Hyperbolic knots with small Seifert-fibered Dehn surgeries, PhD thesis, University of Texas at Austin (1996)

[16] A Deruelle, K Miyazaki, K Motegi, Networking Seifert fibered surgeries on knots, Preprint 
[17] R Fintushel, R J Stern, Constructing lens spaces by surgery on knots, Math. Z. 175 (1980) 33-51 MR595630

[18] H Goda, M Hirasawa, Y Yamada, Lissajous curves as A'Campo divides, torus knots and their fiber surfaces, Tokyo J. Math. 25 (2002) 485-491 MR1948678

[19] H Goda, M Teragaito, Dehn surgeries on knots which yield lens spaces and genera of knots, Math. Proc. Cambridge Philos. Soc. 129 (2000) 501-515 MR1780501

[20] R E Gompf, A I Stipsicz, 4-manifolds and Kirby calculus, Graduate Studies in Math. 20, Amer. Math. Soc. (1999) MR1707327

[21] C M Gordon, Dehn surgery on knots, from: "Proceedings of the International Congress of Mathematicians, Vol. I, II (Kyoto, 1990)”, Math. Soc. Japan, Tokyo (1991) 631-642 MR1159250

[22] C M Gordon, Dehn filling: a survey, from: "Knot theory (Warsaw, 1995)", (V F R Jones, J Kania-Bartoszyn'ska, J H Przytycki, P Traczyk, V G Turaev, editors), Banach Center Publ. 42, Polish Acad. Sci., Warsaw (1998) 129-144 MR1634453

[23] S M Guseĭn-Zade, Intersection matrices for certain singularities of functions of two variables, Funkcional. Anal. i Priložen. 8 (1974) 11-15 MR0338437

[24] J Harer, A Kas, R Kirby, Handlebody decompositions of complex surfaces, Mem. Amer. Math. Soc. 62 (1986) iv+102 MR849942

[25] M Hedden, Notions of positivity and the Ozváth-Szabó concordance invariant arXiv: math.GT/0509.499

[26] M Hedden, On Floer homology and the Berge conjecture on knots admitting lens space surgeries arXiv:math.GT/0710.0357

[27] P Hill, K Murasugi, On double-torus knots. II, J. Knot Theory Ramifications 9 (2000) 617-667 MR1762760

[28] M Hirasawa, Visualization of A'Campo's fibered links and unknotting operation, from: "Proceedings of the First Joint Japan-Mexico Meeting in Topology (Morelia, 1999)", Topology Appl. 121 (2002) 287-304 MR1903697

[29] R Kirby, A calculus for framed links in $S^{3}$, Invent. Math. 45 (1978) 35-56 MR0467753

[30] N Maruyama, On Dehn surgery along a certain family of knots, J. Tsuda College (1987) 261-280 MR892642

[31] K Morimoto, Y Yamada, A note on essential tori in the exterior of torus knots with twists, to appear in Kobe J. Math.

[32] L Moser, Elementary surgery along a torus knot, Pacific J. Math. 38 (1971) 737-745 MR0383406

[33] Y Ni, Knot Floer homology detects fibred knots, Invent. Math. 170 (2007) 577-608 MR2357503 
[34] RP Osborne, RS Stevens, Group presentations corresponding to spines of 3manifolds. III, Trans. Amer. Math. Soc. 234 (1977) 245-251 MR0488063

[35] P Ozsváth, Z Szabó, On knot Floer homology and lens space surgeries, Topology 44 (2005) 1281-1300 MR2168576

[36] C V Quach Hongler, C Weber, The link of an extrovert divide, Ann. Fac. Sci. Toulouse Math. (6) 9 (2000) 133-145 MR1815944

[37] L Rudolph, Knot theory of complex plane curves, from: "Handbook of knot theory", (W Menasco, M Morwen Thistlethwaite, editors), Elsevier B. V., Amsterdam (2005) 349-427 MR2179266

[38] J R Stallings, Constructions of fibred knots and links, from: "Algebraic and geometric topology (Proc. Sympos. Pure Math., Stanford Univ., 1976), Part 2”, (R J Milgram, editor), Proc. Sympos. Pure Math. XXXII, Amer. Math. Soc. (1978) 55-60 MR520522

[39] M Tange, Lens spaces given from L-space homology 3-spheres arXiv: math.GT/0709.0141

[40] M Teragaito, Dehn surgery on knots yielding lens spaces, Lecture, The 7-th JapanKorea school of knots and links (Kobe) (1999)

[41] Y Yamada, Plane slalom curves of a certain type, pretzel links and Kirby-Melvin's Grapes (in Japanese), Sūri-Kaiseki-Kenkyū-Sho Kō-Kyū-Roku

[42] Y Yamada, Berge's knots in the fiber surfaces of genus one, lens space and framed links, J. Knot Theory Ramifications 14 (2005) 177-188 MR2128509

[43] Y Yamada, A family of knots yielding graph manifolds by Dehn surgery, Michigan Math. J. 53 (2005) 683-690 MR2207215

[44] Y Yamada, Finite Dehn surgery along A'Campo's divide knots, from: "Singularity theory and its applications", (S Izumiya, G Ishikawa, H Tokunaga, I Shimada, T Sano, editors), Adv. Stud. Pure Math. 43, Math. Soc. Japan, Tokyo (2006) 573-583 MR2325154

Department of Systems Engineering, University of Electro-Communications 1-5-1, Chofugaoka, Chofu, Tokyo 182-8585, JAPAN

yyyamada@sugaku.e-one.uec.ac.jp

http://mathweb.e-one.uec.ac.jp/ yyyamada/

Received: 29 October 2007 Revised: 10 February 2009 


\begin{tabular}{|c|c|c|c|ll|}
\hline & $a$ & $A$ & $l$ & \multicolumn{1}{|c|}{$\delta, \varepsilon \in\{ \pm 1\}, t \in \mathbf{Z}$} \\
\hline III & 0 & $2,3,4, \ldots$ & $3,5,7, \ldots$ (odd) & $B=A l-\varepsilon, \quad b=-\delta \varepsilon(2 A+t B)$ \\
IV & 1 & $5,7,9, \ldots$ (odd) & $5,7,9, \ldots$ (odd) & $B=(A l-\varepsilon) / 2, b=-\delta \varepsilon(A+t B)$ \\
V & 1 & $3,5,7, \ldots$ (odd) & $2,3,4, \ldots$ & $B=A l+\varepsilon^{*}, \quad b=-\delta \varepsilon(A+t B)$ \\
VI & 0 & $4,6,8, \ldots$ (even) & & $B=2 A+1, \quad b=\delta(A-1+t B)$ \\
\hline
\end{tabular}

Here $\varepsilon^{*} \neq-1$ if $l=2$ (since $\left.0<2 A \leq B\right)$.

(1) The parities and ranges of $A, l$ and the settings of $a, B, b$ (see [11])

\begin{tabular}{rrrr}
\hline III: $l=3+2 k$, & $\mathrm{V}: l=2+k \quad$ (if $\varepsilon=+1)$, & VI: $k \equiv 0 \quad(\varepsilon \equiv-1)$. \\
IV: $l=5+2 k$, & $l=3+k$ (if $\varepsilon=-1)$, & \\
\hline
\end{tabular}

(2) Parameter $k$

$\begin{array}{ll}\text { III: } & -\delta \varepsilon\left(6 A^{2}-3 \varepsilon A+k(2 A)^{2}+t B^{2}\right), \\ \text { IV: } & -\delta \varepsilon\left(\frac{5}{2} A^{2}-\frac{3}{2} \varepsilon A+k A^{2}+t B^{2}\right) \\ \mathrm{V}: & -\delta\left(2 A^{2}+k A^{2}+t B^{2}\right) \text { if } \varepsilon=+1, \\ & \delta\left(3 A^{2}+k A^{2}+t B^{2}\right) \text { if } \varepsilon=-1, \\ \mathrm{VI}: & \delta\left(\left(2 A^{2}-1\right)+t B^{2}\right) .\end{array}$

(3) Surgery coefficient: $b B+\delta A$

\begin{tabular}{|c|c|c|c|c|}
\hline & $A$ & $k$ (if $\varepsilon=+1)$ & $k$ (if $\varepsilon=-1)$ & $\varepsilon p \neq$ \\
\hline III & $n+1$ & $p-1$ & $p-2$ & $-1,0$ \\
IV & $2 n+1$ & $p-2$ & $p-3$ & $-2,-1,0,1$ \\
V & $2 n+3$ & $p-2$ & $p-3$ & $-2,-1,0,1$ \\
VI & $2 n+2$ & - & $(0)$ & - \\
\hline
\end{tabular}

(4) Parameter translation (to $[10 ; 7 ; 9 ; 16]$ ); see Section 6.3.

Table 1: Parameters of Berge knots 
Type III $\varepsilon=1, A=2,3,4, \ldots$

$\varepsilon=-1, A=2,3,4, \ldots$

coef $=6 A^{2}-3 A$, coef $=6 A^{2}+3 A$ $\operatorname{area}(\mathcal{L})=6 A^{2}-3 A$, $\operatorname{area}(\mathcal{L})=6 A^{2}+3 A+1$
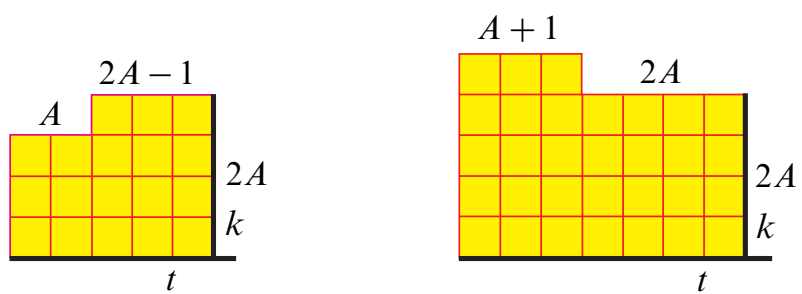

Type IV

$$
\begin{aligned}
& \varepsilon=1, A=5,7,9, \ldots \\
& \text { coef }=\frac{5}{2} A^{2}-\frac{3}{2} A \\
& \operatorname{area}(\mathcal{L})=\frac{5}{2} A^{2}-\frac{3}{2} A+1
\end{aligned}
$$

$$
\begin{aligned}
& \varepsilon=-1, A=5,7,9, \ldots \\
& \text { coef }=\frac{5}{2} A^{2}+\frac{3}{2} A \\
& \operatorname{area}(\mathcal{L})=\frac{5}{2} A^{2}+\frac{3}{2} A
\end{aligned}
$$

$$
(3 A+1) / 2
$$

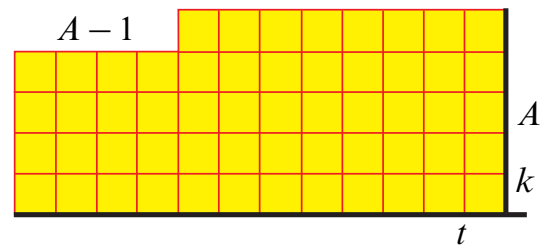

Type V

$$
\begin{aligned}
& \varepsilon=1, A=3,5,7, \ldots \\
& \text { coef }=2 A^{2} \text {, } \\
& \operatorname{area}(\mathcal{L})=2 A^{2}+1 \text {, }
\end{aligned}
$$

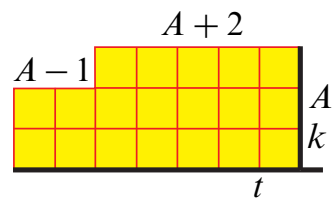

A

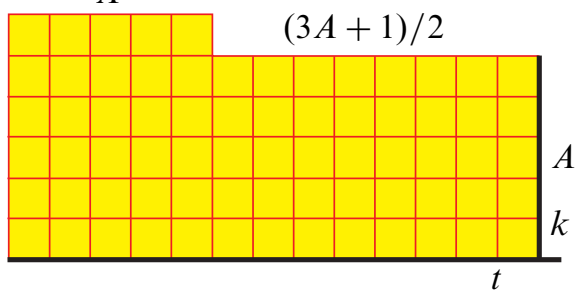

$\varepsilon=-1, A=3,5,7, \ldots$

coef $=3 A^{2}$

$\operatorname{area}(\mathcal{L})=3 A^{2}$

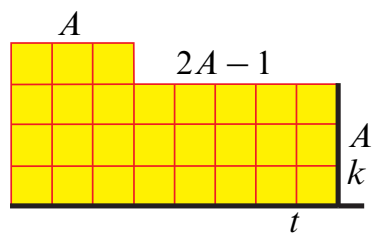

\section{Type VI}

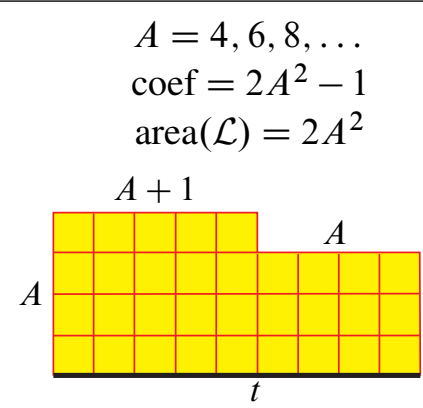

Table 2: Berge knots presented by L-shaped regions 